\title{
Enrichment of Comammox and Nitrite-Oxidizing Nitrospira From Acidic Soils
}

\section{Yu Takahashi', Hirotsugu Fujitani², Yuhei Hirono ${ }^{3}$, Kanako Tago ${ }^{4}$, Yong Wang ${ }^{4}$, Masahito Hayatsu ${ }^{4}$ and Satoshi Tsuneda ${ }^{\text {* }}$}

1 Department of Life Science and Medical Bioscience, School of Advanced Science and Engineering, Waseda University, Tokyo, Japan, ${ }^{2}$ Department of Biological Sciences, Faculty of Science and Engineering, Chuo University, Tokyo, Japan, ${ }^{3}$ Institute of Fruit Tree and Tea Science, National Agriculture and Food Research Organization (NARO), Shimada, Japan, ${ }^{4}$ Institute for Agro-Environmental Sciences, National Agriculture and Food Research Organization (NARO), Tsukuba, Japan

In agricultural soils fertilized with a high amount of ammonium nitrogen, the $\mathrm{pH}$ decreases because of the oxidation of ammonia by nitrifiers. Molecular-based analyses have revealed that members of the genus Nitrospira dominate over other nitrifiers in some acidic soils. However, terrestrial Nitrospira are rarely cultivated and little is known about their ecophysiology. In addition, recent studies discovered a single microbe with the potential to oxidize both ammonia and nitrite (complete ammonia oxidizer; comammox) within Nitrospira, which had been previously recognized as a nitrite oxidizer.

OPEN ACCESS

Edited by: Laura E. Lehtovirta-Morley, University of East Anglia, United Kingdom

Reviewed by: Baozhan Wang,

Nanjing Normal University, China Aqiang Ding,

Chongqing University, China

*Correspondence:

Satoshi Tsuneda

stsuneda@waseda.jp

Specialty section:

This article was submitted to Microbial Physiology and Metabolism, a section of the journal

Frontiers in Microbiology

Received: 25 March 2020

Accepted: 02 July 2020

Published: 22 July 2020

Citation:

Takahashi Y, Fujitani H, Hirono Y,

Tago K, Wang Y, Hayatsu $M$ and

Tsuneda S (2020) Enrichment of Comammox and Nitrite-Oxidizing

Nitrospira From Acidic Soils.

Front. Microbiol. 11:1737.

doi: 10.3389/fmicb.2020.01737
Despite their broad distribution, there are no enrichment samples of comammox from terrestrial or acidic environments. Here, we report the selective enrichment of both comammox and nitrite-oxidizing Nitrospira from the acidic soil of a heavily fertilized tea field. Long-term enrichment was performed with two individual continuous-feeding bioreactors capable of controlling ammonia or nitrite concentration and $\mathrm{pH}$. We found that excessive ammonium supply was a key factor to enhance the growth of comammox Nitrospira under acidic conditions. Additionally, a low concentration of nitrite was fed to prevent the accumulation of free nitrous acid and inhibition of cell growth under low $\mathrm{pH}$, resulting in the selective enrichment of nitrite-oxidizing Nitrospira. Based on $16 \mathrm{~S}$ rRNA gene analysis, Nitrospira accounting for only $1.2 \%$ in an initial soil increased to approximately $80 \%$ of the total microorganisms in both ammonia- and nitrite-fed bioreactors. Furthermore, amoA amplicon sequencing revealed that two phylotypes belonging to comammox clade A were enriched in an ammonia-fed bioreactor. One group was closely related to previously cultivated strains, and the other was classified into a different cluster consisting of only uncultivated representatives. These two groups coexisted in the bioreactor controlled at pH 6.0, but the latter became dominant after the $\mathrm{pH}$ decreased to 5.5. Additionally, a physiological experiment revealed that the enrichment sample oxidizes ammonia at $\mathrm{pH}<4$, which is in accordance with the strongly acidic tea field soil; this value is lower than the active $\mathrm{pH}$ range of isolated acid-adapted nitrifiers. In conclusion, we successfully enriched multiple phylotypes of comammox and nitrite-oxidizing Nitrospira and revealed that the $\mathrm{pH}$ and concentrations of protonated $\mathrm{N}$-compounds were potential niche determinants.

Keywords: acidic soil, nitrifying bacteria, nitrification, Nitrospira, comammox, cultivation, enrichment, low pH 


\section{INTRODUCTION}

Nitrification is an important reaction composing the global nitrogen cycle. In soil ecosystems, nitrification acts as a production route of nitrate, a nitrogen source of soil plants. Despite the importance of nitrification, supplementation with excessive nitrogen fertilizers over-activates nitrification and induces environmental problems. Too much nitrate produced by nitrification leaches into groundwater, causing pollution and the economic loss of fertilizers (Schlesinger, 2009). Furthermore, excessive nitrification also causes the release of nitrous oxide, a greenhouse gas (Wrage et al., 2001) and soil acidification (Vitousek et al., 1997). In particular, acidic soils (defined as $\mathrm{pH}$ $<5.5)$ are known to show the same or higher nitrification rate as neutral soils (Booth et al., 2005). Moreover, soils with lower $\mathrm{pH}$ were reported to generate more nitrous oxide (Mørkved et al., 2007; Liu et al., 2010; Bakken et al., 2012).

Traditionally, nitrification had been thought to consist of two independent steps: ammonia oxidation $\left(\mathrm{NH}_{3}\right.$ to $\left.\mathrm{NO}_{2}{ }^{-}\right)$ and nitrite oxidation $\left(\mathrm{NO}_{2}{ }^{-}\right.$to $\left.\mathrm{NO}_{3}{ }^{-}\right)$. The first step is catalyzed by ammonia-oxidizing bacteria (AOB) and ammoniaoxidizing archaea (AOA), while the second step is carried out by nitrite-oxidizing bacteria (NOB). Molecular approaches revealed that AOA predominated among ammonia oxidizers in soils (Leininger et al., 2006) including acidic soils (Nicol et al., 2008; Zhang et al., 2012). In addition, a strain of acidophilic AOA, "Candidatus Nitrosotalea devanaterra" was isolated from acidic soil (Lehtovirta-Morley et al., 2011) and physiologically characterized (Lehtovirta-Morley et al., 2014). Although these studies supported the importance of AOA in nitrification in acidic soils, $\mathrm{AOB}$ was reported to outnumber $\mathrm{AOA}$ in some acidic soils (Long et al., 2012; Petersen et al., 2012; Wertz et al., 2012). Furthermore, a strain of acid-tolerant AOB, "Candidatus Nitrosoglobus terrae" was isolated from the acidic soil of a tea field (Hayatsu et al., 2017). This study proved that the phylogenetically novel AOB classified into Gammaproteobacteria were more abundant than other ammonia oxidizers in some tea fields (Hayatsu et al., 2017). In contrast, for acidophilic NOB, Nitrobacter sp. Io acid was isolated from acidic forest soil (Hankinson and Schmidt, 1988). All of the acid-tolerant isolates of NOB were classified into the genus Nitrobacter: Nitrobacter sp. Io acid (Hankinson and Schmidt, 1988), Nitrobacter winogradskyi (Bock and Heinrich, 1969), and Nitrobacter strain NHB1, cocultured with AOB (De Boer et al., 1991). However, Nitrospira, a different genus of NOB, outnumbers Nitrobacter in other acidic soils (Wertz et al., 2012; Stempfhuber et al., 2017). Moreover, in several studies cultivating samples from wastewater treatment plants and soilless medium-based horticulture systems, Nitrospira became dominant in acidic cultures at $\mathrm{pH}<5$ (Tarre and Green, 2004; Cytryn et al., 2012). Although these cultures were not from soils, such previous reports support that uncultivated Nitrospira could contribute to nitrification in acidic environments.

Recent studies found a complete ammonia oxidizer (comammox) in the genus Nitrospira; this novel bacterium oxidizes both ammonia and nitrite in a single cell (Daims et al., 2015; van Kessel et al., 2015). Comammox Nitrospira is phylogenetically diverse and separated into two sister clades named as clade $\mathrm{A}$ and $\mathrm{B}$, based on ammonia monooxygenase subunit A (amoA) gene sequence (Daims et al., 2015). Since this discovery, many studies have investigated the potential metabolisms and environmental distributions of comammox based on molecular biological techniques such as metagenomics (Palomo et al., 2016, 2018; Bartelme et al., 2017; Camejo et al., 2017; Wang et al., 2017; Orellana et al., 2018). Based on these metagenomic datasets, quantitative PCR primers targeting comammox were designed (Pjevac et al., 2017). In a study using this primer set, comammox was reported to be the most dominant in nitrifying bacteria communities in acidic soils at $\mathrm{pH}$ 4.0-7.0 ( $\mathrm{Hu}$ and $\mathrm{He}, 2017)$. While the importance of comammox and nitrite-oxidizing Nitrospira in acidic soils has been clarified as described above, cultivation of these bacteria has hardly been attempted. Only one study obtained an isolate of comammox, Nitrospira inopinata, from a biofilm sustained in thermal waters at pH 7.5 (Daims et al., 2015; Dimitri et al., 2017). Also, "Candidatus Nitrospira nitrosa" and "Candidatus Nitrospira nitrificans" were enriched from a recirculation aquaculture system biofilter in a sequencing batch reactor operated at $\mathrm{pH} 6.99$ (van Kessel et al., 2015). Other culture samples of comammox were obtained from wastewater treatment plants (Camejo et al., 2017; Roots et al., 2019) nitrifying granules (Fujitani et al., 2013), and river sediments (Yu et al., 2018). On the basis of these cultivation researches and genomic studies, physiological and biochemical characteristics of comammox Nitrospira have been speculated. Comammox Nitrospira is presumed to have advantage over other nitrifiers under low dissolved oxygen concentration and adapted to slow growth in oligotrophic environments (Koch et al., 2019). However, all the comammox cultures were incubated with a neutral or alkaline medium. For this reason, culture samples allowing the study of the acid-adaptation and ecology of comammox Nitrospira in acidic soils have not been obtained yet.

In this study, we focused on the cultivation of comammox and nitrite-oxidizing Nitrospira from acidic soils. The soil sample was collected from a tea field. Tea fields are supplemented with higher amount of $\mathrm{N}$ fertilizer than typical croplands and the excessive fertilization causes $\mathrm{N}$-related problems, such as nitrate leaching (Hirono et al., 2009), soil acidification, and $\mathrm{N}_{2} \mathrm{O}$ production (Tokuda and Hayatsu, 2004). Using two types of bioreactors, we enriched both comammox and nitrite-oxidizing Nitrospira at $\mathrm{pH}$ 5.5. During a long-term enrichment process over 2 years, the microbial community was analyzed by $16 \mathrm{~S}$ rRNA gene and amoA gene amplicon sequencing. Furthermore, ammonia oxidation activity tests using the enrichment samples were performed to investigate adaptation to different $\mathrm{pH}$ and ammonia concentrations.

\section{MATERIALS AND METHODS}

\section{Soil Samples}

Following our previous study (Hayatsu et al., 2017) soil samples were collected in August 2017 from tea field plots supplemented with $\mathrm{N}$ fertilizer of $506 \mathrm{~kg} \mathrm{~N} \mathrm{ha}^{-1}$ year $^{-1}$ at Kanaya Tea Research 
Station, Institute of Fruit Tree and Tea Science, NARO, in Japan $\left(34^{\circ} 48^{\prime} 28.2^{\prime \prime} \mathrm{N} 138^{\circ} 07^{\prime} 55.9^{\prime \prime} \mathrm{E}\right)$. Soil samples were immediately stored at $-80^{\circ} \mathrm{C}$ or $4^{\circ} \mathrm{C}$. Molecular analyses were performed on the samples stored at $-80^{\circ} \mathrm{C}$. The characteristics of the soils are shown in Supplementary Table S1. Samples stored at $4^{\circ} \mathrm{C}$ were used for incubation within a month of sampling.

\section{Continuous-Feeding Incubation}

Following our previous research (Fujitani et al., 2013) two types of continuous-feeding bioreactors were set up to selectively enrich comammox and nitrite-oxidizing Nitrospira. A $13 \mathrm{~g}$ soil sample was suspended in an inorganic medium and incubated in batch culture. As biomass carriers for soil bacteria, nonwoven fabrics were sunk in the medium. To attach the biomass to the non-woven fabrics, the suspended soil sample was statically incubated without exchanging the medium. The $\mathrm{pH}$ in the medium was manually kept under 6.0 and the concentration of $\mathrm{NH}_{4} \mathrm{Cl}$ in the medium was kept under $0.4 \mathrm{mM}$. After pre-incubation for a month, the non-woven fabrics and supernatant in the batch culture were transferred to a bioreactor. Subsequently, inorganic medium containing $\mathrm{NH}_{4} \mathrm{Cl}$ was continuously supplied into the continuous-feeding bioreactor (day 0 of continuous-feeding incubation). The biomass was maintained on the non-woven fabrics to prevent microorganisms to be washed out from the bioreactor. On day 25, part of the biomass in the $\mathrm{NH}_{4} \mathrm{Cl}$-fed bioreactor was transplanted to the other bioreactor, and a medium containing $\mathrm{NaNO}_{2}$ was supplied continuously.

The components of inorganic medium supplied into the $\mathrm{NH}_{4} \mathrm{Cl}$-fed and $\mathrm{NaNO}_{2}$-fed bioreactors are shown in Supplementary Tables S2, S3, respectively. The capacity of both bioreactors was $1.0 \mathrm{~L}$ and the inorganic media were supplied at a rate of $3.0 \mathrm{~L} \mathrm{day}^{-1}$. The bioreactors were operated in a dark room maintained at $23^{\circ} \mathrm{C}$ and were supplied with excess oxygen by aeration. The inorganic media and $\mathrm{pH}$ adjustment chemicals were supplied by tubing pumps (ATTO Co., Tokyo, Japan) into the bioreactors. The influx of $\mathrm{pH}$ adjustment chemicals was automatically operated by digital $\mathrm{pH}$ controllers (Nissin Rika Co., Tokyo, Japan), which are able to measure the $\mathrm{pH}$ values in the bioreactors and control the flux in real time. $\mathrm{NaHCO}_{3}$ and $\mathrm{HCl}$ were used as $\mathrm{pH}$ adjustment chemicals for the $\mathrm{NH}_{4} \mathrm{Cl}$ - and $\mathrm{NaNO}_{2}$-fed bioreactors, respectively.

During continuous-feeding incubation of the $\mathrm{NH}_{4} \mathrm{Cl}$-fed bioreactor, the $\mathrm{NH}_{4} \mathrm{Cl}$ concentration in the supplied medium increased in a stepwise way from 0.07 to $0.14,0.71,1.4,2.1$, $7.1,18,29,39$, and $50 \mathrm{mM}$ (day 0-206). The inflow $\mathrm{NH}_{4} \mathrm{Cl}$ concentration was determined, following our previous study (Hayatsu et al., 2017). From day 206 to the end of the experiment, the concentration was maintained at $50 \mathrm{mM}$. The $\mathrm{pH}$ in the $\mathrm{NH}_{4} \mathrm{Cl}$-fed bioreactor was controlled at $\mathrm{pH} 6.0 \pm 0.2$ from day 0 to 373 . On day 374 , after the stable nitrification was observed at $\mathrm{pH} 6.0$, the $\mathrm{pH}$ value was decreased to $5.5 \pm 0.2$, and kept a constant level until the end of the experiment. Likewise, the concentration of $\mathrm{NaNO}_{2}$ in the medium supplied into the $\mathrm{NaNO}_{2}$-fed bioreactor was increased in a stepwise manner from 0.07 to $0.14,0.29,0.43,0.71,1.8,2.9$, and $3.9 \mathrm{mM}$ (day 25-150), following our previous research (Fujitani et al., 2013). Thereafter, the concentration was maintained for a while, but was again reduced from 3.9 to $1.8,0.71$, and $0.36 \mathrm{mM}$ at last from day 310 to 387 to reduce the stress on the biomass. The $\mathrm{pH}$ in the $\mathrm{NaNO}_{2}-$ fed bioreactor was controlled at $\mathrm{pH} 6.0 \pm 0.2$ from day 0 to 303 . The $\mathrm{pH}$ value was decreased to $5.5 \pm 0.2$ on day 304 and kept at a constant level until the end of the experiment.

\section{Chemical Analyses During Continuous-Feeding Incubation}

For each culture condition, the culture solution in each bioreactor was collected and sterilized by passing through $0.22 \mu \mathrm{m}$ polyethersulfone membrane filters (Millipore, Eschborn, Germany). These solution samples were stored at $-20^{\circ} \mathrm{C}$ until chemical analyses to measure the total ammonianitrogen $\left(\mathrm{NH}_{4}{ }^{+}+\mathrm{NH}_{3}\right)$, nitrite-nitrogen $\left(\mathrm{NO}_{2}{ }^{-} \mathrm{-N}\right)$, and nitrate-nitrogen $\left(\mathrm{NO}_{3}{ }^{-}-\mathrm{N}\right)$ concentrations. The total ammonia-nitrogen concentration was measured using the indophenol blue method (Kandeler and Gerber, 1988) with a PowerWave HT microplate spectrophotometer (BioTek Instruments Inc., Winooski, VT, United States) using the absorbance at $630 \mathrm{~nm}$ as an index. The nitrite-nitrogen and nitrate-nitrogen concentrations were measured using an IC-2010 ion chromatography system (Tosoh Co., Tokyo, Japan). Based on the measured values, the concentrations of free ammonia $\left(\mathrm{NH}_{3}\right)$ and free nitrite $\left(\mathrm{HNO}_{2}\right)$ were calculated according to the method of a previous study (Anthonisen et al., 1976).

\section{Microscopic Observation}

The cell suspensions were collected from non-woven fabrics in bioreactors on days $84,228,319$, and 791 . The samples were fixed and stored at $-20^{\circ} \mathrm{C}$ until observation. The fixed samples were sonicated with a Q55 homogenizer (Qsonica LLC., Newtown, CT, United States) at 20\% amplitude for 30 $s$ to disperse bacterial aggregates. The sonicated samples were dropped onto slide glasses and stained using fluorescence in situ hybridization (FISH) as described in a previous study (Amann et al., 1990). Oligonucleotide probes binding specifically to $16 \mathrm{~S}$ rRNA of Nitrospira lineage II, Nitrospira lineage I, Nitrobacter, and betaproteobacterial AOB were labeled with hydrophilic sulfoindocyanine dye (Су3) (Supplementary Table S4). These probes were added to the slide glasses and hybridized at $46^{\circ} \mathrm{C}$ for $2.5 \mathrm{~h}$. Furthermore, all bacteria were stained with SYTOX Green nucleic acid stain (Life Technologies, Carlsbad, CA, United States). The stained samples were observed with an Axioskop 2 Plus fluorescence microscope (Carl Zeiss, Oberkochen, Germany).

\section{DNA Extraction}

Genomic DNA was extracted from the initial soil samples used for cultivation and cell suspensions collected from the bioreactors. The extraction was performed using the FastDNA SPIN Kit for Soil (MP Biomedicals, Irvine, CA, United States) according to the protocol of the manufacturer. The extracted DNA was stored at $-20^{\circ} \mathrm{C}$ until amplicon sequencing. 


\section{Amplicon Sequencing of 16S rRNA Gene and amoA Gene}

Amplicon sequencing targeting the variable regions $\mathrm{V} 7$ and V8 of the 16S rRNA gene was performed with primers with added adapter sequences (Supplementary Table S5). The extracted genomic DNA was amplified by PCR using Ex Taq (Takara Bio Inc., Shiga, Japan). The amplicon was sequenced by the Ion Torrent Personal Genome Machine sequencer (Life Technologies, Carlsbad, CA, United States) (McDonald et al., 2012). Sequence data were processed with CLC Genomics Workbench v5.5.1 (CLC bio, Aarhus, Denmark). The barcode sequences, sequences of inappropriate length $(<250$ bp or $>350 \mathrm{bp}$ ), sequences with low-quality scores (limit $=0.005$ ), and sequences containing ambiguous nucleotides (five nucleotides at most) were trimmed. The processed sequences were analyzed using QIIME (Caporaso et al., 2010) and sequences with 98\% or more homology were compiled as operational taxonomic units (OTUs) using the UCLUST algorithm (Edgar, 2010). One representative sequence was extracted from each OTU, and the sequences were assigned referring to the SILVA database version 132 (Quast et al., 2013). Sequences sharing less than $80 \%$ homology with the references were defined as unclassified. To investigate the phylogeny of OTUs in more detail, sequences of OTUs classified as nitrifiers were analyzed using the Basic Local Alignment Search Tool (BLAST) server ${ }^{1}$ of the National Center for Biotechnology Information (NCBI).

Likewise, adapter sequences were added to a primer pair targeting the amoA gene of comammox (Supplementary Table S5). PCR amplification, sequencing, and trimming procedures were the same as those for the 16S rRNA gene, except for trimming and compiling. The sequences of inappropriate length ( $<150 \mathrm{bp}$ or $>250 \mathrm{bp})$ were trimmed and sequences with homology of $97 \%$ or more were summarized as OTUs.

\section{Phylogenetic Analyses}

The sequences of $16 \mathrm{~S}$ rRNA genes classified into each lineage of the genus Nitrospira were searched on the nucleotide database of $\mathrm{NCBI}^{2}$. Based on these sequences and representative sequences of OTUs obtained in this study, phylogenetic analysis was performed using MEGA7 software (Shindo et al., 2006). The collected sequences were aligned and a phylogenetic tree was constructed using the neighbor-joining method (Saitou and Nei, 1987) and the bipartition confidence was evaluated at 1,000 bootstraps (Felsenstein, 1985).

The whole genome sequences of cultivated comammox, metagenome-assembled genomes (MAGs) constructed from environmental samples and clone sequences of the Nitrospira amoA gene detected in cultured samples were searched for in the NCBI nucleotide database, and AmoA amino acid sequences were collected. In case coding sequences (CDSs) of amoA were not annotated in the GenBank entry on the database, the MAG sequences were downloaded and CDSs were annotated using Prokka (version 1.13) (Seemann, 2014). To obtain AmoA amino

${ }^{1}$ https://blast.ncbi.nlm.nih.gov/Blast.cgi

${ }^{2}$ https://www.ncbi.nlm.nih.gov/nucleotide/ acid sequences of enriched comammox and to correct the frameshift of those sequences, the nucleotide sequences of OTUs were translated using the FrameBot tool (Wang et al., 2013) referring to the AmoA sequence of Nitrospira inopinata. The phylogenetic tree based on AmoA amino acid sequences was constructed using the same method as that for the $16 \mathrm{~S}$ rRNA genes.

\section{Nitrification Experiments of $\mathrm{NH}_{4} \mathrm{Cl}-\mathrm{Fed}$ Enrichment}

To examine the effect of $\mathrm{NH}_{4} \mathrm{Cl}$ concentration on the nitrification activity, a batch culture test was performed. An enrichment sample was collected from the $\mathrm{NH}_{4} \mathrm{Cl}$-fed bioreactor on day 749 and washed using centrifugation $(2,900 \times g, 10 \mathrm{~min})$. The fresh inorganic medium described above was added to the sample and the bacterial pellet was dispersed with a Q55 homogenizer (Qsonica LLC, Newtown, CT, United States) for $30 \mathrm{~s}$ at $20 \%$ amplitude. The suspended sample was transferred into test tubes containing inorganic medium adjusted to $\mathrm{pH} 5.5$, containing 0 , $12.5,25,50,75,100,200$, or $300 \mathrm{mM} \mathrm{NH}_{4} \mathrm{Cl}$. The test tubes were incubated for three days in a dark room maintained at $23^{\circ} \mathrm{C}$. The test was performed with three biological replicates. The supernatant of the medium in the tubes was collected and stored at $-20^{\circ} \mathrm{C}$ until chemical analyses.

To examine the effect of $\mathrm{pH}$ on nitrification activity, another batch culture test was performed. The method for preparing bacterial suspension was as described above, except for the sampling was performed on day 849 . The sample was transferred into test tubes with inorganic medium containing $12.5 \mathrm{mM}$ $\mathrm{NH}_{4} \mathrm{Cl}$, adjusted to $\mathrm{pH} 3.0,4.0,5.0,6.4,7.5$, or 9.0. As buffers of the medium, $80 \mathrm{mM}$ 2-morpholinoethanesulfonic acid (MES) for $\mathrm{pH}$ 5.0, 4-(2-hydroxyethyl)-1-piperazine-ethanesulphonic acid (HEPES) for $\mathrm{pH}$ 6.4, and $25 \mathrm{mM} \mathrm{N}$-cyclohexyl-3aminopropanesulfonic acid (CAPS) for $\mathrm{pH} 7.5$ and 9.0 were mixed. The concentration of CAPS was adjusted to the condition described in a previous report (Dai et al., 2012). The media for the $\mathrm{pH} 3.0$ and 4.0 tests were unbuffered. The tubes were incubated for 2 days and collected samples were stored as described above.

\section{Chemical Analyses for Nitrification Experiments}

The nitrite concentrations of culture samples were measured using the Griess test (Griess-Romijn, 1966) using the absorbance at $560 \mathrm{~nm}$ as the index. As described in a previous study (Miranda et al., 2001) nitrate concentrations were determined by reducing nitrate to nitrite with vanadium chloride (III) and measured using the Griess test. The absorbance was measured using a PowerWave HT microplate spectrophotometer (BioTek Instruments Inc., Winooski, VT, United States).

\section{RESULTS AND DISCUSSION}

\section{Water Quality During Continuous-Feeding Incubation}

At the start of the continuous-feeding incubation in the $\mathrm{NH}_{4} \mathrm{Cl}-$ fed bioreactor, the medium was maintained at around $\mathrm{pH}$ 6.0. 


\section{The $\mathrm{NH}_{4} \mathrm{Cl}$-fed bioreactor}

A

$\mathrm{NH}_{4}{ }^{+}+\mathrm{NH}_{3}, \mathrm{NO}_{2}^{-}$and $\mathrm{NO}_{3}{ }^{-}$concentrations in the $\mathrm{NH}_{4} \mathrm{Cl}$-fed bioreactor

- Influent total ammonia $\left(\mathrm{NH}_{4}{ }^{+}+\mathrm{NH}_{3}\right)$ concentration [mM]

$\rightarrow$ Total ammonia $\left(\mathrm{NH}_{4}{ }^{+}+\mathrm{NH}_{3}\right)$ concentration in the bioreactor [mM]

$\rightarrow$ Nitrite $\left(\mathrm{NO}_{2}{ }^{-}\right)$concentration in the bioreactor [mM]

$\rightarrow$ Nitrate $\left(\mathrm{NO}_{3}{ }^{-}\right)$concentration in the bioreactor [mM]

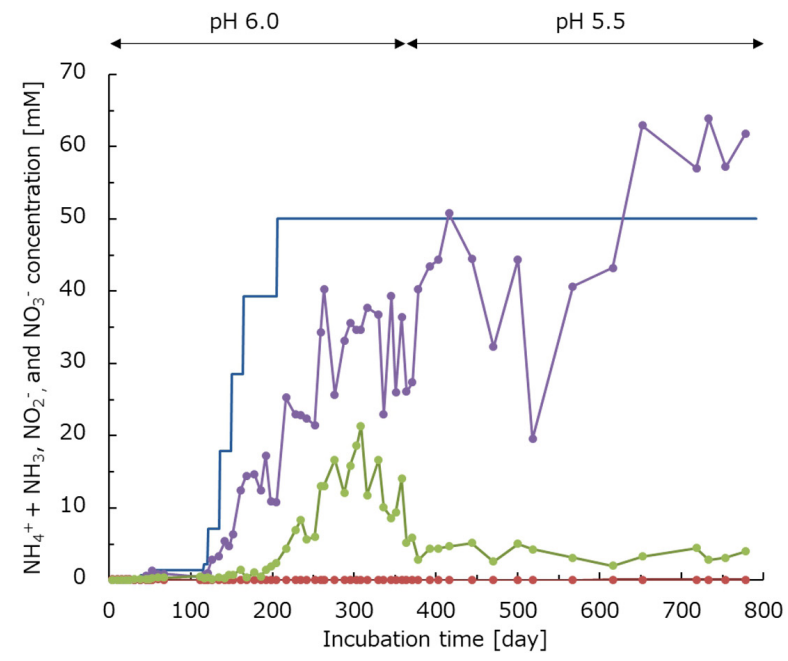

C

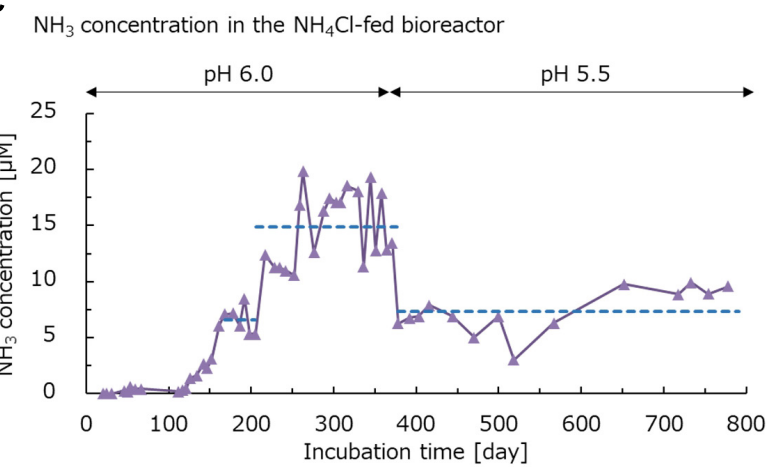

The $\mathrm{NaNO}_{2}$-fed bioreactor

B

$\mathrm{NO}_{2}{ }^{-}$and $\mathrm{NO}_{3} \cdot$ concentrations in the $\mathrm{NaNO}_{2}$-fed bioreactor

- Influent nitrite $\left(\mathrm{NO}_{2}{ }^{-}\right)$concentration $[\mathrm{mM}]$

$\rightarrow-$ Nitrite $\left(\mathrm{NO}_{2}^{-}\right)$concentration in the bioreactor $[\mathrm{mM}]$

$\rightarrow-$ Nitrate $\left(\mathrm{NO}_{3}{ }^{-}\right)$concentration in the bioreactor $[\mathrm{mM}]$

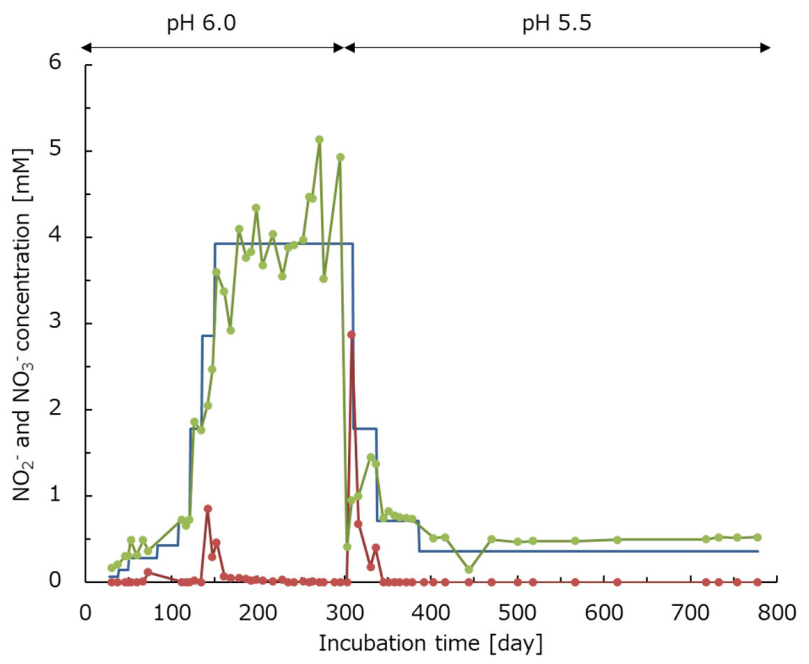

D

$\mathrm{HNO}_{2}$ concentration in the $\mathrm{NaNO}_{2}$-fed bioreactor

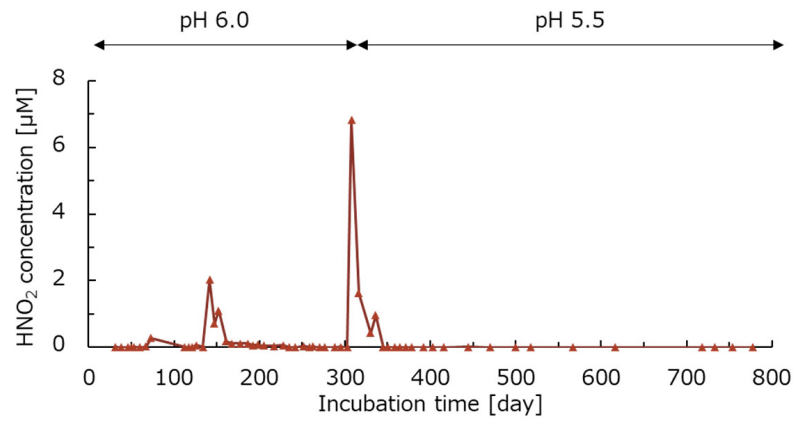

FIGURE 1 | Total ammonia, nitrite, and nitrate concentrations in the (A) $\mathrm{NH}_{4} \mathrm{Cl}$-fed bioreactor and (B) $\mathrm{NaNO}_{2}$-fed bioreactor. The theoretically calculated concentration of $\mathrm{NH}_{3}$ in the $\mathrm{NH}_{4} \mathrm{Cl}$-fed bioreactor and $\mathrm{HNO}_{2}$ in the $\mathrm{NaNO}_{2}$-fed bioreactor are shown in (C,D), respectively. The dashed lines in (C) show the average value of each period. The $\mathrm{pH}$ values in the $\mathrm{NH}_{4} \mathrm{Cl}$-fed bioreactor and $\mathrm{NaNO}_{2}$-fed bioreactor were changed from 6.0 to 5.5 on days 374 and 304 , respectively.

Under acidic conditions, the concentration of free ammonia $\left(\mathrm{NH}_{3}\right)$ decreased exponentially as the $\mathrm{pH}$ decreased, owing to protonation from ammonia to ammonium $\left(\mathrm{NH}_{4}{ }^{+}\right)(\mathrm{pKa}=9.25)$ (De Boer and Kowalchuk, 2001). Ammonia, not ammonium, is known as the substrate of ammonia monooxygenase (Suzuki et al., 1974; Galloway et al., 2008). Considering the decrease in ammonia concentration caused by protonation, an excessive amount of ammonium was supplied into the bioreactor to enrich the comammox Nitrospira. The concentration of $\mathrm{NH}_{4} \mathrm{Cl}$ in the supplied medium was increased in a stepwise way. On day 165-205, when the bioreactor was supplied with the medium containing $39 \mathrm{mM} \mathrm{NH}_{4} \mathrm{Cl}$, the nitrate $\left(\mathrm{NO}_{3}{ }^{-}\right)$concentration in the bioreactor increased to $1.3 \pm 0.8 \mathrm{mM}$ on average, which shows an increase in nitrification activity (Figure 1A). After the further increase in supplied $\mathrm{NH}_{4} \mathrm{Cl}$ concentration to $50 \mathrm{mM}$, the $\mathrm{NO}_{3}{ }^{-}$concentration in the bioreactor reached $11.2 \pm 4.9 \mathrm{mM}$ during days 206-373. Although the total ammonia $\left(\mathrm{NH}_{4}{ }^{+}+\right.$ $\mathrm{NH}_{3}$ ) in the bioreactor was not completely oxidized to nitrate, a low concentration of $\mathrm{NO}_{3}{ }^{-}$was stably produced by nitrification. Based on this result, the influent concentration of $\mathrm{NH}_{4} \mathrm{Cl}$ was fixed at $50 \mathrm{mM}$ in the subsequent incubation. To enrich the nitrifiers adapted to a more acidic condition, we decreased the $\mathrm{pH}$ from 6.0 to 5.5 , in which most of the isolated ammonia oxidizers cannot grow (De Boer and Kowalchuk, 2001). After the decrease in $\mathrm{pH}$ during days 374-791, the $\mathrm{NO}_{3}{ }^{-}$concentration in the bioreactor reduced to $3.7 \pm 1.0 \mathrm{mM}$, indicating the suppression of nitrification activity. However, in acidic soils, high concentration of ammonium accumulated (Supplementary 
Table S1). On the other hand, nitrate concentration in soils was two orders of magnitude less than that of ammonium. Thus, the culture condition with low $\mathrm{pH}$ and high influent concentration of ammonia could reproduce the environment in acidic soils, although ammonia in the bioreactor was not completely oxidized. Furthermore, the concentration of $\mathrm{NO}_{2}{ }^{-}$was maintained at under $0.2 \mathrm{mM}$ throughout the cultivation process. This result was also in accordance with the low nitrite concentration in soils (Kowalchuk and Stephen, 2001).Throughout the incubation, the measured concentration of total ammonia $\left(\mathrm{NH}_{4}{ }^{+}+\mathrm{NH}_{3}\right)$ in the bioreactor was not stable. This is considered to be due to the high reagent blank of the indophenol blue method (Willason and Johnson, 1986; Aminot et al., 1997; Kérouel and Aminot, 1997). Moreover, the stoichiometric ratio of ammonia consumption and nitrate generation was not consistent. This inconsistency could be caused by the consumption of ammonia as nitrogen source or denitrification converting nitrate to gaseous nitrogen.

A part of the biomass in the $\mathrm{NH}_{4} \mathrm{Cl}$-fed bioreactor was transferred to the $\mathrm{NaNO}_{2}$-fed bioreactor on day 25. The medium in the bioreactor was maintained at around $\mathrm{pH}$ 6.0. Under low $\mathrm{pH}$ conditions, the concentration of free nitrous acid $\left(\mathrm{HNO}_{2}\right)$ increases because of the protonation of $\mathrm{NO}_{2}{ }^{-}(\mathrm{pKa}=3.3)$. Considering that even low concentrations of $\mathrm{HNO}_{2}$ suppress nitrification (Anthonisen et al., 1976) the concentration of $\mathrm{NO}_{2}{ }^{-}$ in the bioreactor was controlled to be as low as possible to avoid the accumulation of $\mathrm{HNO}_{2}$. The concentration of $\mathrm{NaNO}_{2}$ in the supplied medium was increased in a stepwise way but controlled carefully to completely oxidize all $\mathrm{NO}_{2}{ }^{-}$in the bioreactor. Therefore, $\mathrm{NO}_{2}{ }^{-}$was completely oxidized to $\mathrm{NO}_{3}{ }^{-}$ and the $\mathrm{NO}_{3}{ }^{-}$concentration in the bioreactor increased as the influent concentration of $\mathrm{NO}_{2}{ }^{-}$increased during days 28-303 (Figure 1B). On day 304, the $\mathrm{pH}$ was reduced from 6.0 to 5.5. Therefore, the concentration of $\mathrm{NO}_{2}{ }^{-}$in the bioreactor rapidly increased to $2.9 \mathrm{mM}$ on day 308 . The decrease in nitrite oxidation activity could be due to the increase in $\mathrm{HNO}_{2}$ with the change in $\mathrm{pH}$. To avoid the accumulation of $\mathrm{HNO}_{2}$ in the bioreactor, we step-wisely reduced the $\mathrm{NaNO}_{2}$ concentration in the supplied medium from $3.93 \mathrm{mM}$ to $0.36 \mathrm{mM}$ during days $310-387$. Just after decreasing the inflow $\mathrm{NO}_{2}{ }^{-}$concentration from $3.93 \mathrm{mM}$ to $1.79 \mathrm{mM}, \mathrm{NO}_{3}{ }^{-}$concentration in the bioreactor partially recovered. Furthermore, as the $\mathrm{NO}_{2}{ }^{-}$concentration in the inflow medium decreased, the $\mathrm{NO}_{2}{ }^{-}$concentration in the bioreactor gradually decreased to reach $0 \mathrm{mM}$. These results suggest that the nitrite-oxidation activity in the $\mathrm{NaNO}_{2}$-fed bioreactor was recovered by adjusting the $\mathrm{NO}_{2}{ }^{-}$concentration in the supplied medium, although the oxidation was temporarily inhibited by a decrease in $\mathrm{pH}$. Therefore, we presumed that the sharp decrease in the nitrite oxidation rate could be caused not only by the change in $\mathrm{pH}$, but also by the increase in $\mathrm{HNO}_{2}$ concentration.

To investigate the relationship between the nitrification activity and the concentration of $\mathrm{NH}_{3}$ or $\mathrm{HNO}_{2}$, the theoretical concentration of $\mathrm{NH}_{3}$ in the $\mathrm{NH}_{4} \mathrm{Cl}$-fed bioreactor and $\mathrm{HNO}_{2}$ concentration in the $\mathrm{NaNO}_{2}$-fed bioreactor were calculated according to the method of a previous study (Anthonisen et al., 1976). In the $\mathrm{NH}_{4} \mathrm{Cl}$-fed bioreactor controlled at $\mathrm{pH} 6.0$ until day 373, the $\mathrm{NH}_{3}$ concentration in the bioreactor increased as the concentration of $\mathrm{NH}_{4} \mathrm{Cl}$ in the supplied medium increased
(Figure 1C). When the medium containing $39 \mathrm{mM} \mathrm{NH}_{4} \mathrm{Cl}$ was supplied during days 165-205, the average $\mathrm{NH}_{3}$ concentration was $6.6 \pm 1.2 \mu \mathrm{M}$. Afterward, the $\mathrm{NH}_{4} \mathrm{Cl}$ concentration in the supplied medium increased to $50 \mathrm{mM}$ on day 206 and the average $\mathrm{NH}_{3}$ concentration in the bioreactor increased to $14.9 \pm 3.2 \mu \mathrm{M}$ during days 206-373. On day 374, the $\mathrm{pH}$ was decreased from 6.0 to 5.5; concurrently the $\mathrm{NH}_{3}$ concentration also decreased and kept constant at $7.4 \pm 2.0 \mu \mathrm{M}$ until day 791. Based on this result, the decrease in the nitrification rate after $\mathrm{pH}$ change is considered to be partly caused by the decreasing concentration of free ammonia, the substrate for nitrification.

Furthermore, in the $\mathrm{NaNO}_{2}$-fed bioreactor controlled at $\mathrm{pH}$ 6.0, $\mathrm{HNO}_{2}$ temporarily increased up to $2.0 \mu \mathrm{M}$ on day 142 (Figure 1D). This was due to the sudden increase in the influent $\mathrm{NO}_{2}{ }^{-}$concentration. However, at this point, the nitrification rate increased rapidly and the $\mathrm{NO}_{2}{ }^{-}$concentration in the bioreactor decreased immediately by day 161 . From this result, the nitrifier community in the bioreactor would be still active under the conditions of $1.1 \mu \mathrm{M} \mathrm{HNO}_{2}$ on day 161. In contrast, the nitrification activity decreased immediately after the $\mathrm{pH}$ was reduced from 6.0 to 5.5 on day 304. Simultaneously, the $\mathrm{HNO}_{2}$ concentration increased to $6.8 \mu \mathrm{M}$ on day 308 and remained in the bioreactor until day 336. During this period, nitrification in the bioreactor did not recover unless the influent $\mathrm{NO}_{2}{ }^{-}$ concentration was reduced. Although $6.8 \mu \mathrm{M}$ of $\mathrm{HNO}_{2}$ inhibited nitrification in the bioreactor, the nitrifier community would be still active under $\mathrm{pH}$ 5.5. Consequently, it is considered that $\mathrm{HNO}_{2}$, rather than low $\mathrm{pH}$, suppressed the nitrification activity.

\section{Morphology of Nitrifiers}

The non-woven fabrics in both bioreactors became brown colored with the biomass (Supplementary Figure S1). The cell suspensions collected from the bioreactors formed flocs visible to naked eyes. The morphological feature of these flocs was similar to an enrichment culture of Nitrospira in a previous report (Spieck et al., 2006).

In the $\mathrm{NH}_{4} \mathrm{Cl}$-fed bioreactor, Nitrospira lineage II and betaproteobacterial AOB grew remarkably (Figure 2A). On day 84, several cells of Nitrospira lineage II aggregated and formed small colonies. Such small colonies gathered to form larger dense aggregates on day 228 and 319. Until day 319, those aggregates were mainly composed of Nitrospira lineage II cells. However, on day 791, Nitrospira lineage II were co-aggregated with colonies of other bacteria. This morphological change in Nitrospira lineage II might be caused by the $\mathrm{pH}$ decrease. The co-aggregates containing Nitrospira lineage II resembled the structures observed in the culture incubating " $\mathrm{Ca}$. Nitrospira nitrosa" and "Ca. Nitrospira nitrificans" (van Kessel et al., 2015) rather than the small microcolonies of Nitrospira inopinata (Daims et al., 2015). In contrast, betaproteobacterial AOB grew throughout the incubation. As in Nitrospira lineage II, aggregates mainly composed of AOB cells were observed on day 319 at $\mathrm{pH}$ 6.0. However, AOB adhered to other bacteria on day 791 at $\mathrm{pH}$ 5.5. Such a co-aggregation of betaproteobacterial AOB with other bacteria was induced by $\mathrm{pH}$ reduction in a previous study (De Boer et al., 1991). Unlike the significant growth of Nitrospira 


\section{The $\mathrm{NH}_{4} \mathrm{Cl}$-fed bioreacto}

A Nitrifiers in the $\mathrm{NH}_{4} \mathrm{Cl}$-fed bioreactor

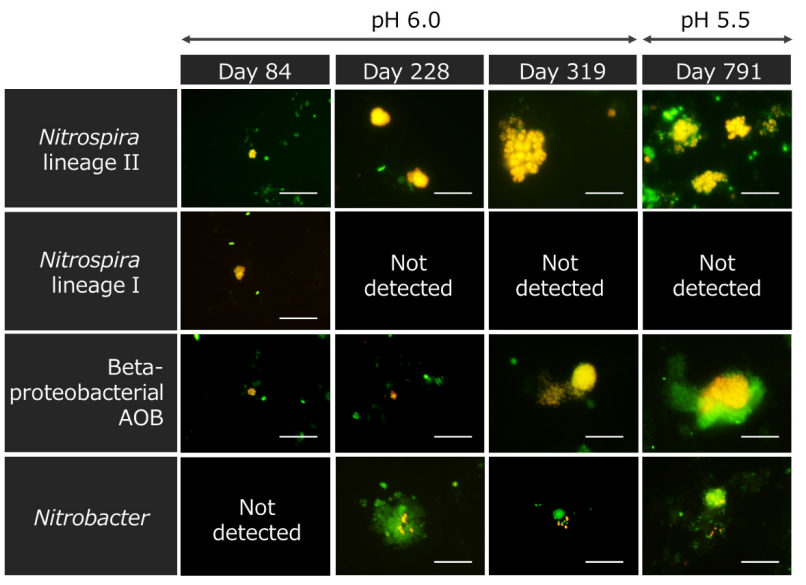

The $\mathrm{NaNO}_{2}$-fed bioreactor

B Nitrifiers in the $\mathrm{NaNO}_{2}$-fed bioreactor

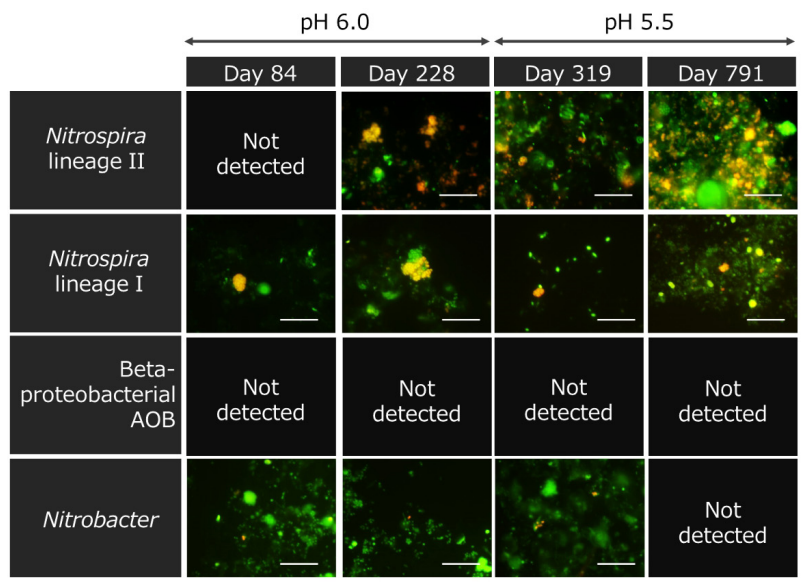

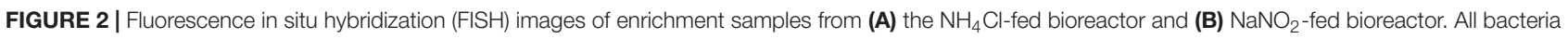
were stained with SYTOX Green (green). Only targeted cells were labeled with FISH probes (red) and two colors were merged into yellow. The scale bars represent $10 \mu \mathrm{m}$.

lineage II and AOB, Nitrospira lineage I and Nitrobacter were hardly observed throughout the incubation.

Bacteria in the $\mathrm{NaNO}_{2}$-fed bioreactor formed strongly aggregated structures with widths of several tens of micrometers (Figure 2B). On day 228, after the stepwise increase in the influent $\mathrm{NaNO}_{2}$ concentration, dozens of Nitrospira lineage II cells formed colonies. On day 319, just after changing the $\mathrm{pH}$ from 6.0 to 5.5, Nitrospira lineage II decreased and its colonies became smaller. However, on day 791, after the $\mathrm{NaNO}_{2}$ concentration in the bioreactor was kept low, Nitrospira lineage II grew again. At $\mathrm{pH} 5.5$, Nitrospira lineage II aggregated with other bacteria and formed a complex structure. This aggregation is considered to be an adaptation to lower $\mathrm{pH}$ or higher concentrations of $\mathrm{HNO}_{2}$. Nitrospira was reported to increase the production of extracellular polymeric substances to form aggregates under high nitrite concentrations (Nowka et al., 2015). The structure of Nitrospira lineage I aggregates was similar to that of lineage II. Furthermore, betaproteobacterial AOB was under the detection limit of microscopic observation throughout the incubation. In addition, only a small number of Nitrobacter cells were observed as a part of the aggregates.

\section{S rRNA Gene-Based Bacterial Community}

Based on the V7-V8 region of the 16S rRNA gene, the bacterial community structure in the bioreactors was analyzed at the family level. In the initial soil sample, the family Nitrospiraceae including comammox accounted for $1.2 \%$ of the total bacteria, which was the largest among nitrifying bacteria (Figure 3). In contrast, the family Nitrosomonadaceae including betaproteobacterial $\mathrm{AOB}$ and the family Bradyrhizobiaceae including the genus Nitrobacter occupied $<0.1 \%$ of the total bacteria in the initial soil sample.
In the $\mathrm{NH}_{4} \mathrm{Cl}$-fed bioreactor, the nitrification activity increased during days 165-205 when the medium containing $39 \mathrm{mM} \mathrm{NH} 4 \mathrm{Cl}$ was supplied. At the same time, the relative abundance of family Nitrospiraceae also increased and reached $60.1 \%$ by day 206 (Figure 3A). After that, the abundance of Nitrospiraceae fluctuated within 55.8-92.9\%, but consistently accounted for more than half of the whole bacterial population, regardless of the $\mathrm{pH}$ change. Although another nitrifier oxidizing ammonia, Nitrosomonadaceae also increased in number, the abundance remained $<8.4 \%$ throughout the incubation. The relative abundance of Bradyrhizobiaceae including Nitrobacter also remained $<7.3 \%$. Considering that a small number of AOB existed and a small number of $\mathrm{NO}_{2}{ }^{-}$were produced in the bioreactor, some bacteria of the family Nitrospiraceae would function as Nitrospira oxidizing only $\mathrm{NO}_{2}{ }^{-}$and not $\mathrm{NH}_{3}$. However, based on the great difference between the proportion of Nitrosomonadaceae and that of Nitrospiraceae, most of the Nitrospiraceae in the bioreactor was assumed to function as comammox Nitrospira oxidizing $\mathrm{NH}_{3}$ to $\mathrm{NO}_{3}{ }^{-}$. Since the family Nitrospiraceae became dominant in the bioreactor and maintained a stable proportion, the culture condition in this study was considered to be suitable for cultivating Nitrospiraceae from an acidic soil. In addition, an isolate of acidophilic AOA from acidic soils was reported to be deactivated at pH 6.0 (Lehtovirta-Morley et al., 2014). Thus, the initial culture condition at $\mathrm{pH} 6.0$ might eliminate AOA in the soils. However, we did not try to detect AOA in the enrichment sample with molecular biological techniques such as quantitative PCR. Therefore, note that the possibility of ammonia oxidation by AOA cannot be denied. Moreover, even though Nitrospiraceae was enriched with inorganic medium, some heterotrophic bacteria such as Rhodanobacteraceae remained in the bioreactor. Rhodanobacteraceae is known as a heterotrophic denitrifying bacterium (Kostka et al., 2012). Those bacteria might consume 


\section{The $\mathrm{NH}_{4} \mathrm{Cl}$-fed bioreactor}

A Bacterial community structure in the $\mathrm{NH}_{4} \mathrm{Cl}$-fed bioreactor

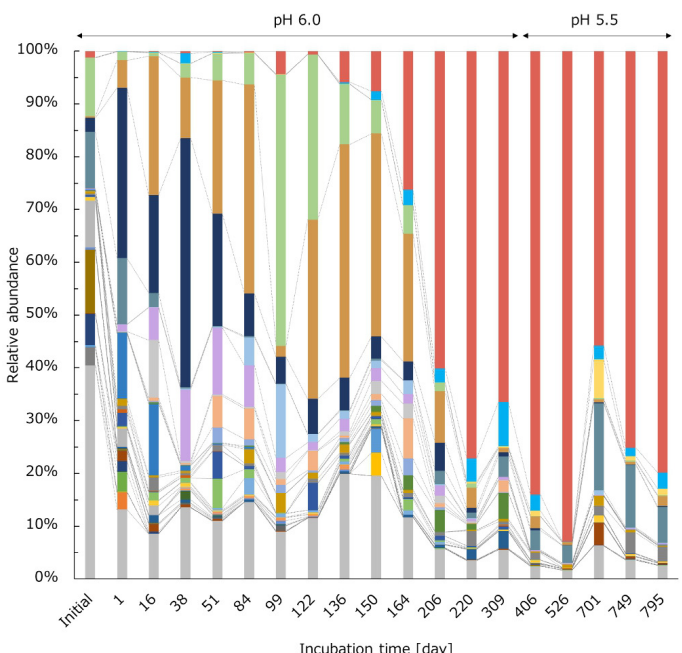

C Community structure of Nitrospiraceae in the $\mathrm{NH}_{4} \mathrm{Cl}$-fed bioreactor

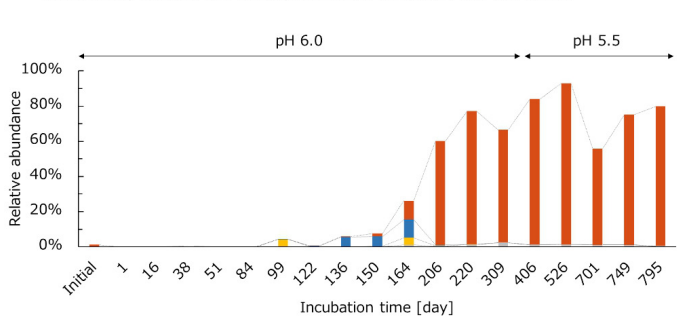

The $\mathrm{NaNO}_{2}$-fed bioreactor

B Bacterial community structure in the $\mathrm{NaNO}_{2}$-fed bioreactor

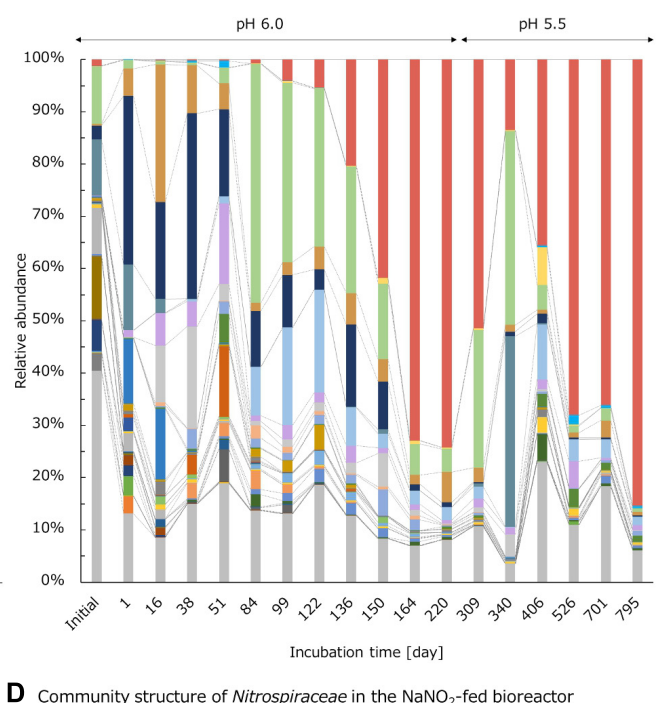

= Nitrospiraceae

Nitrosomonadaceae

- Bradyrhizobiaceae

Nocardiaceae

- Burkholderiaceae

- Rhodanobacteracea

= Reyranellaceae

Mphingomonadaceae
Pseudomonadaceae

= Rickettsiaceae

= Simkaniaceae

- Hyphomonadacea

Pedosphaeraceae

- Chitinophagaceae

- Rhodocyclaceae

- unassigned Saccharimonadales - Paracaedibacteraceae - unassigned Thermomicrobiales Xanthobacteraceae

Acetobacteraceae

- Gemmataceae

- Gemmataceae

- unassigned Flavobacteriales

- Acidothermaceae

- Opitutaceae

- Rhizobiaceae

- Acidobacteriacea

- Alcanivoracaceae

- Enterobacteriaceae

Midichloriaceae

- Parvibaculaceae

unassigned or rare groups

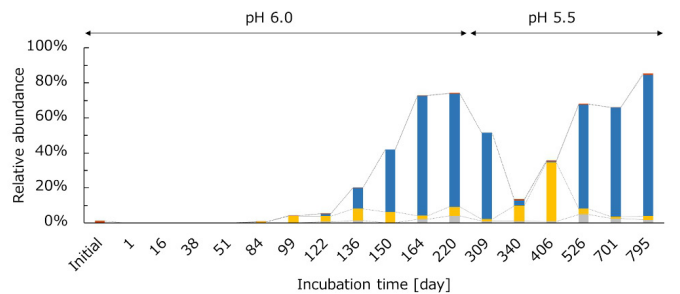

— Soil Nitrospira $16 \mathrm{~S}$ OTU1 - Soil Nitrospira $16 \mathrm{~S}$ OTU2 = Soil Nitrospira $16 \mathrm{~S}$ OTU3 n Rare OTUs

FIGURE 3 | Taxonomic assignments of 16S rRNA gene sequences of enrichment cultures in (A,C) the $\mathrm{NH}_{4} \mathrm{Cl}$-fed bioreactor and (B,D) $\mathrm{NaNO}_{2}$-fed bioreactor. (A,B) Relative abundances of taxonomic groups were analyzed at the family classification level. Unassigned taxon and phylogenetic groups occupying $<3 \%$ of the total bacteria in all samples were integrated to "unassigned or rare groups." (C,D) Relative abundances of taxonomic groups in the family Nitrospiraceae were analyzed at the OTU level. OTUs occupying < $2 \%$ of the total bacteria in all samples were integrated into "rare OTUs."

$\mathrm{NH}_{3}$ as nitrogen source or reduce $\mathrm{NO}_{3}{ }^{-}$by denitrification to contribute to the inconsistency of stoichiometric ratio of ammonia consumption and nitrate generation (Figure 1A).

Likewise, in the $\mathrm{NaNO}_{2}$-fed bioreactor controlled at $\mathrm{pH}$ 6.0 , the relative abundance of Nitrospiraceae increased as the influent $\mathrm{NO}_{2}{ }^{-}$concentration gradually increased up to $3.93 \mathrm{mM}$ during days 28-150. After that, the $\mathrm{pH}$ in the bioreactor and influent $\mathrm{NO}_{2}{ }^{-}$concentration kept a constant level until day 303 . In this period, the relative abundance of family Nitrospiraceae increased to $72.9 \%$ on day 164 and remained an almost constant abundance until day 220 (Figure 3B). However, the nitrification activity of the bioreactor decreased after the $\mathrm{pH}$ was decreased from 6.0 to 5.5 on day 304. Concurrently, the proportion of Nitrospiraceae decreased from $51.4 \%$ on day 309 to $13.5 \%$ on day 340. In contrast, the abundance of the family Bradyrhizobiaceae, including Nitrobacter, increased from $0.32 \%$ on day 309 to $7.20 \%$ on day 406 following the $\mathrm{pH}$ change. This different response between Nitrospira and Nitrobacter to $\mathrm{pH}$ change is considered to be due to the relatively higher resistance of Nitrobacter to $\mathrm{HNO}_{2}$ (Blackburne et al., 2007). After the decrease in $\mathrm{HNO}_{2}$ concentration in the bioreactor, the nitrification was reactivated and the relative abundance of Nitrospiraceae recovered to $35.6 \%$ on day 406. During days 526-795, the bioreactor operated under a stable environment, and the abundance of Nitrospiraceae reached to the range of $66.1 \%-85.4 \%$. In the environment with a low concentration of $\mathrm{HNO}_{2}$, however, the relative abundance of Bradyrhizobiaceae was always $<1 \%$, much lower than that of Nitrospiraceae. From this viewpoint, Nitrospira was assumed to be the main contributor to $\mathrm{NO}_{2}{ }^{-}$oxidation in the bioreactor at $\mathrm{pH}$ 5.5 , except for under high concentrations of $\mathrm{HNO}_{2}$. In addition, despite the absence of ammonium in the inflow medium supplied to the bioreactor, the family Nitrosomonadaceae consisting of $\mathrm{AOB}$ grew and comprised $1.82 \%$ of the bacterial community on day 526. Although $\mathrm{AOB}$ needs $\mathrm{NH}_{3}$ as a substrate to grow, most of them are known to produce $\mathrm{NH}_{3}$ by degrading urea (Koper et al., 2004; Norton et al., 2008). Therefore, AOB in the bioreactor might degrade the small amount of urea provided by bacteria coexisting in the bioreactor, which was a limited proportion of the biomass.

Furthermore, community structures of the families Nitrospiraceae, Nitrosomonadaceae, and Bradyrhizobiaceae were analyzed at the level of OTUs. Nitrospiraceae was mainly composed of three OTUs. Before the cultivation, the most abundant of the three OTUs was soil Nitrospira 16S OTU1, 
which accounted for $1.0 \%$ of the whole bacterial community in the initial soil sample (Figure 3C). Considering that the relative proportion of Nitrospiraceae was $1.19 \%$ and the total abundance of Nitrospiraceae, Nitrosomonadaceae, and Bradyrhizobiaceae was only $1.25 \%$, soil Nitrospira 16 S OTU1 was regarded as the dominant bacteria in the community of nitrifiers in the initial soil sample. In the $\mathrm{NH}_{4} \mathrm{Cl}$-fed bioreactor, soil Nitrospira $16 \mathrm{~S}$ OTU1, OTU2, and OTU3 coexisted until day 164, however, soil Nitrospira 16S OTU1 was enriched and accounted for more than half of the bacterial community during days 206-795 (Figure 3C). Based on this result, the continuous-feeding bioreactor supplied with an inorganic medium containing excessive $\mathrm{NH}_{4} \mathrm{Cl}$ at $\mathrm{pH}$ 5.5-6.0 seemed to be suitable for enrichment of the major group of Nitrospira in acidic soil. On the other hand, the other two OTUs were enriched in the $\mathrm{NaNO}_{2}$-fed bioreactor. Soil Nitrospira 16S OTU2, accounting for only $0.02 \%$ in the initial soil, became dominant in the bioreactor on day 164 (Figure 3D). In contrast, the relative abundance of soil Nitrospira $16 \mathrm{~S}$ OTU3 was lower than $7 \%$ throughout the incubation except under high $\mathrm{HNO}_{2}$ concentrations. After the $\mathrm{pH}$ change and the accumulation of $\mathrm{HNO}_{2}$ in the bioreactor, soil Nitrospira $16 \mathrm{~S}$ OTU3 increased its proportion to $8.7 \%$ and $33.8 \%$ on days 340 and 406, respectively. Afterward, the soil Nitrospira 16S OTU3 decreased and soil Nitrospira 16S OTU2 became dominant in the bioreactor again. From this result, the factor deciding the niche differentiation between soil Nitrospira 16S OTU2 and OTU3 would be the tolerance to $\mathrm{HNO}_{2}$, rather than the $\mathrm{pH}$.

The composition of the family Nitrosomonadaceae was also analyzed at the OTU level. The cultivated Nitrosomonadaceae was mainly composed of two OTUs. In the $\mathrm{NH}_{4} \mathrm{Cl}$-fed bioreactor, soil Nitrosospira 16S OTU1 classified into the genus Nitrosospira was enriched (Supplementary Figure S2A). This OTU accounted for $0.011 \%$ of the total bacterial community in the initial soil and was the dominant OTU of the family Nitrosomonadaceae accounting for $0.032 \%$. In the $\mathrm{NaNO}_{2}$-fed bioreactor, soil Nitrosospira 16S OTU2 was enriched, but this group was not detected in the initial soil sample (Supplementary Figure S2B). In contrast to Nitrosomonadaceae, the communities of family Bradyrhizobiaceae in the two bioreactors were composed of a common OTU assigned to the genus Nitrobacter (Supplementary Figure S3). This OTU, named soil Nitrobacter 16S OTU1, was the major OTU in Bradyrhizobiaceae throughout the cultivation and accounted for $0.032 \%$ of the total bacterial community in the initial soil.

\section{$16 S$ rRNA Gene-Based Phylogenetic Analyses}

A phylogenetic tree of Nitrospira based on the 16S rRNA gene sequence was constructed referring to OTUs retrieved from amplicon sequencing and sequences registered in the NCBI nucleotide database. Therefore, soil Nitrospira 16S OTU1 and OTU2 were classified into Nitrospira lineage II (Figure 4). Soil Nitrospira 16S OTU1 was grouped within the cluster including "Candidatus Nitrospira nitrificans" (van Kessel et al., 2015), an enriched strain of comammox Nitrospira, while soil Nitrospira 16S OTU2 was closely related to Nitrospira japonica, an isolated strain of nitrite-oxidizing Nitrospira (Ushiki et al., 2013). On the other hand, soil Nitrospira 16S OTU3 was classified into lineage I and was closely related to Nitrospira sp. ND1 (Fujitani et al., 2013).

In the $\mathrm{NaNO}_{2}$-fed bioreactor, soil Nitrospira 16S OTU2 classified into lineage II and soil Nitrospira 16S OTU3 classified into lineage I competed with one another during the continuousfeeding incubation (Figure 3D). Soil Nitrospira 16S OTU2 did not decrease its relative abundance with exposure to $2.03 \mu \mathrm{M} \mathrm{HNO}_{2}$ during days 142-152, but decreased just after being exposed to $6.8 \mu \mathrm{M} \mathrm{HNO}_{2}$ on day 308 (Figures 1D, 3D); meanwhile, the relative abundance of soil Nitrospira 16S OTU3 increased. From these results, Nitrospira lineage II in the bioreactor was presumed to be advantageous under low concentrations of $\mathrm{NO}_{2}{ }^{-}$regardless of $\mathrm{pH}$ change; however, it was more sensitive to $\mathrm{HNO}_{2}$ than lineage I. By contrast, lineage I was estimated to be favorable under relatively high concentrations of $\mathrm{HNO}_{2}$ and tolerated $6.8 \mu \mathrm{M} \mathrm{HNO}_{2}$ (Table 1). Moreover, a similar niche differentiation between the two lineages was reported in previous studies cultivating Nitrospira from activated sludge under different $\mathrm{NO}_{2}{ }^{-}$concentrations (Maixner et al., 2006; Fujitani et al., 2013). Furthermore, the concentration of diluted oxygen (Park and Noguera, 2008) and usability of organic substrates (Gruber-Dorninger et al., 2015) were reported as the niche differentiation factors between the two lineages. However, all of these theories are based on experiments using samples from wastewater treatment systems. Therefore, the enrichment sample obtained in this study would provide important information to reveal the factors influencing the niche differentiation between the two lineages in acidic conditions and soil environments.

While Nitrospira lineage I contains only nitrite-oxidizing Nitrospira, lineage II also includes comammox. Soil Nitrospira $16 \mathrm{~S}$ OTU1 was enriched in the $\mathrm{NH}_{4} \mathrm{Cl}$-fed bioreactor but was rarely detected in the $\mathrm{NaNO}_{2}$-fed bioreactor (Figure 3). Furthermore, according to metagenomic studies, comammox Nitrospira are known to lack assimilatory nitrite reductase or cyanate hydratase genes for generating ammonia, while nitriteoxidizing Nitrospira do contain these genes and are able to grow in inorganic media containing only $\mathrm{NO}_{2}{ }^{-}$as a nitrogen source (Palomo et al., 2018). In contrast, most of the comammox genomes harbor urease genes to produce ammonia through urea degradation (Palomo et al., 2018), which might enable the growth of comammox depending on urea produced by other bacteria. However, urea-dependent ammonia oxidation is known to take longer than ammonia oxidation under $\mathrm{NH}_{4} \mathrm{Cl}$ supplementation (Pommerening-Roser and Koops, 2005). Based on these facts, soil Nitrospira 16S OTU1 could be composed of comammox Nitrospira. In contrast, soil Nitrospira 16S OTU2 enriched in the $\mathrm{NaNO}_{2}$-fed bioreactor was thought to mainly include nitrite-oxidizing Nitrospira. However, note that the phylogenetic position does not directly support the function of complete ammonia oxidation (Pjevac et al., 2017).

To investigate the phylogenetical positions of OTUs classified into the genera Nitrosospira and Nitrobacter, the related sequences were searched using NCBI BLAST. Soil Nitrosospira 16 S OTU1 was $100 \%$ identical to the $16 \mathrm{~S}$ rRNA gene sequence of Nitrosospira lacus strain APG3. This strain was reported 


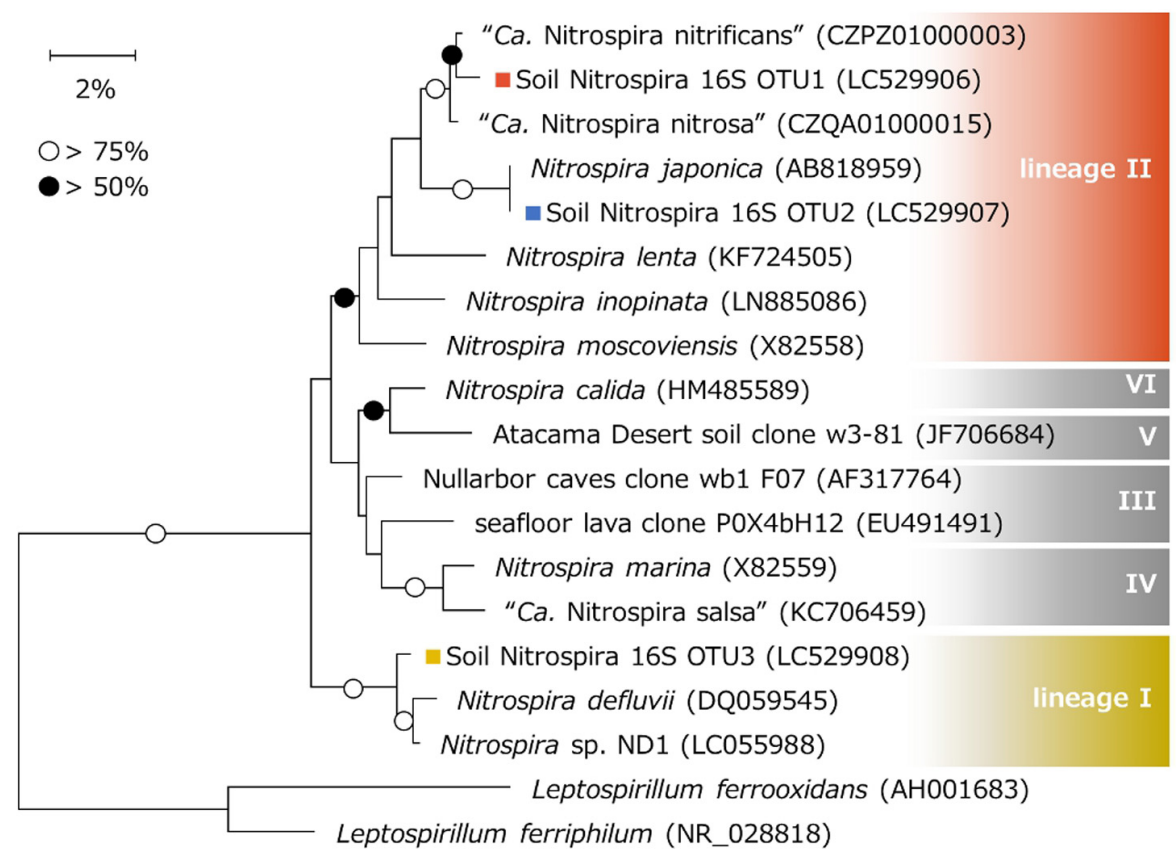

FIGURE 4 | Phylogenetic tree based on the 16S rRNA gene sequences of Nitrospira. Phylogenetic analysis was performed using the neighbor-joining method with 1000 bootstraps. Bootstrap values of $>75 \%$ or $>50 \%$ are indicated as open or closed circles, respectively. Sequences of Leptospirillum were used to root the tree. Colored squares show the OTUs obtained in this study.

TABLE 1 | Features of the OTUs obtained from amplicon sequencing of the 16S rRNA gene and comammox amoA gene.

\begin{tabular}{|c|c|c|c|c|c|c|c|}
\hline \multirow[t]{2}{*}{ OTU } & \multicolumn{3}{|c|}{ Soil Nitrospira $16 \mathrm{~S}$ OTUs } & \multicolumn{4}{|c|}{ Soil Nitrospira amoA OTUs } \\
\hline & OTU1 & OTU2 & оTU3 & OTU1 & оTU3 & OTU4 & OTU2 \\
\hline Source bioreactor & $\mathrm{NH}_{4} \mathrm{Cl}$-fed & \multicolumn{2}{|c|}{$\mathrm{NaNO}_{2}$-fed } & \multicolumn{4}{|c|}{$\mathrm{NH}_{4} \mathrm{Cl}$-fed } \\
\hline Major function & Comammox & \multicolumn{2}{|c|}{ Nitrite oxidation } & \multicolumn{4}{|c|}{ Comammox } \\
\hline Phylogenetic group ${ }^{a}$ & & & lineage I & \multirow{2}{*}{\multicolumn{3}{|c|}{$\begin{array}{c}\text { Uncultivated } \\
\qquad \mathrm{pH} 5.5\end{array}$}} & \multirow{3}{*}{$\begin{array}{c}\text { Cultivated } \\
\mathrm{pH} 6.0\end{array}$} \\
\hline The minimum tolerable $\mathrm{pH}^{\mathrm{b}}$ & \multicolumn{3}{|c|}{$\mathrm{pH} 5.5$} & & & & \\
\hline Tolerable $\mathrm{HNO}_{2}$ concentration ${ }^{\mathrm{C}}$ & \multicolumn{3}{|c|}{ Low } & \multirow{2}{*}{\multicolumn{3}{|c|}{ Low }} & \\
\hline Favorable $\mathrm{NH}_{3}$ concentration ${ }^{d}$ & & & & & & & High \\
\hline
\end{tabular}

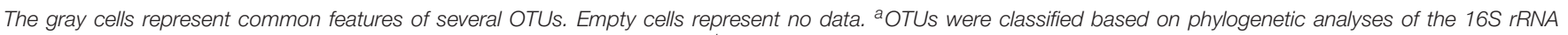

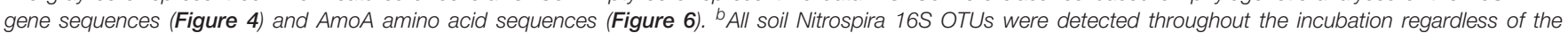

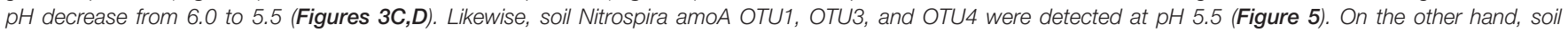

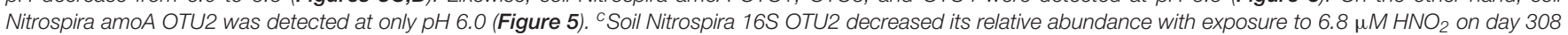

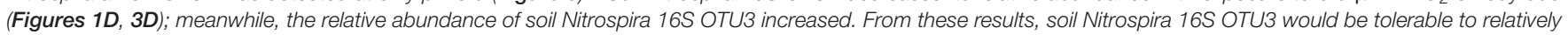

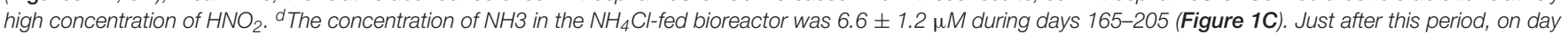

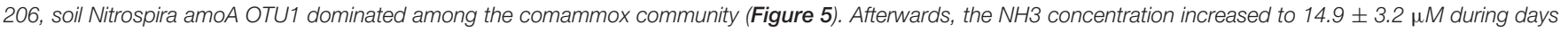

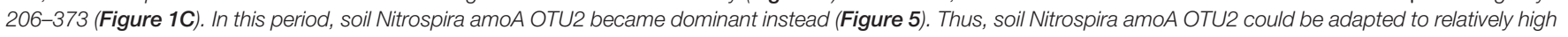
NH3 concentration.

to grow under a wide range of $\mathrm{pH}$ values (5-9) (Urakawa et al., 2015). Although APG3 was isolated from a freshwater lake (Garcia et al., 2013), closely related Nitrosospira was also detected in a soil (Zhang et al., 2015). In contrast, soil Nitrosospira 16S OTU2 cultivated in the $\mathrm{NaNO}_{2}$-fed bioreactor was closely related to Nitrosospira sp. EnI299 enriched from fertilized soil (Tourna et al., 2010). Moreover, soil Nitrobacter 16S OTU1 was $100 \%$ identical to the $16 \mathrm{~S}$ rRNA gene sequence of Nitrobacter sp. Io acid and Nitrobacter vulgaris. Nitrobacter sp. Io acid was isolated from an acidic forest soil and showed nitrite-oxidizing activity at $\mathrm{pH}$ 3.5-7.0 (Hankinson and Schmidt, 1988). Nitrobacter vulgaris DSM10236 was cultivated in media with neutral or mildly alkaline $\mathrm{pH}$, such as 7.4 or 8.6 (Reshetilov et al., 2011). For a different strain of the same species, Nitrobacter vulgaris strain mesi survived in a fairly wide range of $\mathrm{pH}$ values (6.0-9.2) (Laanbroek and Schotman, 1991). Therefore, the adaptable $\mathrm{pH}$ range for Nitrobacter depends on each strain and it is difficult to distinguish the range based solely on the 16S rRNA gene sequence at this point. 


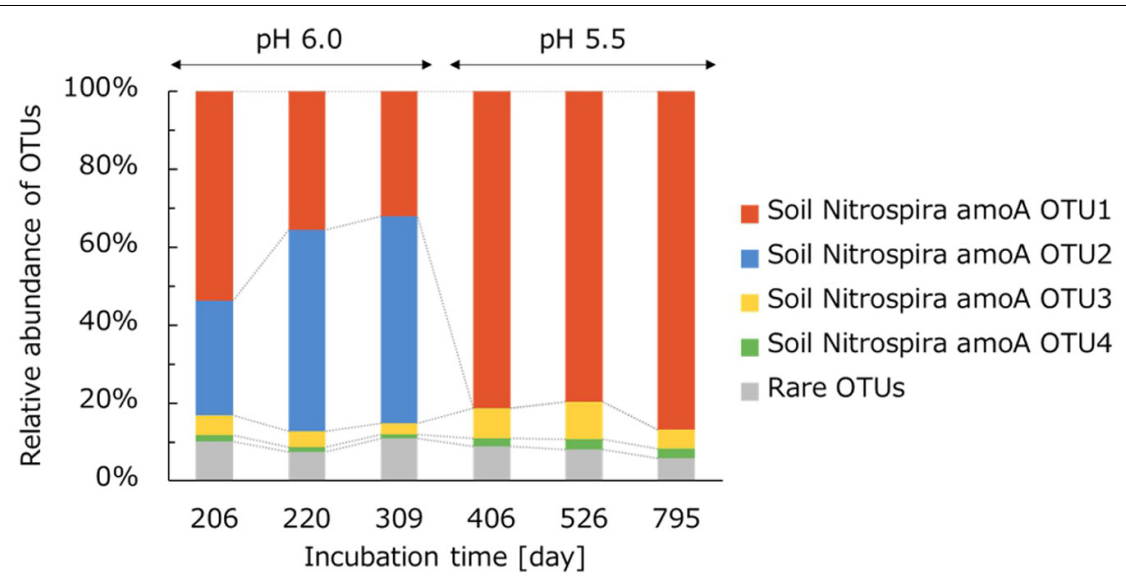

FIGURE 5 | Relative abundance of taxonomic groups in comammox Nitrospira in the $\mathrm{NH}_{4} \mathrm{Cl}$-fed bioreactor. Taxonomic groups were analyzed based on comammox amoA gene sequences at the OTU classification level. OTUs occupying $<2 \%$ of the total comammox community in all samples were integrated into "rare OTUs."

\section{amoA Gene-Based Bacterial Community of Comammox Nitrospira}

As described above, Nitrospira lineage II includes both comammox and nitrite-oxidizing Nitrospira; it is difficult to distinguish these two groups based on $16 \mathrm{~S}$ rRNA gene sequences (Pjevac et al., 2017). To analyze the phylogenetic features of comammox Nitrospira specifically, an amplicon sequencing targeted at the comammox amoA gene was performed. However, the comammox amoA gene in the extracted DNA samples was not amplified before day 206 (data not shown). The impossibility of amplification could be due to the low abundance of comammox Nitrospira and low PCR efficiency of barcodeattached primers. The relative abundance of each OTU was calculated as a proportion to the total reads of comammox amoA sequences. The comammox Nitrospira community in the $\mathrm{NH}_{4} \mathrm{Cl}$-fed bioreactor was mainly composed of four OTUs. Other OTUs accounting for less than $2 \%$ of all samples were integrated into "rare OTUs." Especially, two OTUs, named soil Nitrospira amoA OTU1 and soil Nitrospira amoA OTU2 were dominant (Figure 5). During days 206-309, the $\mathrm{pH}$ in the bioreactor was controlled at 6.0 and the concentration of $\mathrm{NH}_{4} \mathrm{Cl}$ in the influx medium was maintained at $50 \mathrm{mM}$. In this period, the relative abundance of soil Nitrospira amoA OTU2 slowly increased, while that of soil Nitrospira amoA OTU1 decreased (Figure 5). After the $\mathrm{pH}$ change from 6.0 to 5.5, however, soil Nitrospira amoA OTU2 was not detected and soil Nitrospira amoA OTU1 became dominant instead.

The dynamics of the relative abundances of comammox OTUs caused by the $\mathrm{pH}$ change indicated that the soil Nitrospira amoA OTU2 activity was suppressed or completely inhibited at $\mathrm{pH}$ 5.5. The acidic condition stresses microorganisms by affecting $\mathrm{pH}$ homeostasis or decreasing $\mathrm{HCO}_{3}{ }^{-}$as a carbon source (Lehtovirta-Morley et al., 2016; Herbold et al., 2017). Thus, the $\mathrm{pH}$ decrease might suppress the growth of Nitrospira amoA OTU2. The concentration of $\mathrm{NH}_{3}$ in the bioreactor controlled at $\mathrm{pH} 5.5$ during days $374-791$ corresponded to the value at pH 6.0 during days 165-205 (Figure 1C). Just after this period, on day 206, soil Nitrospira amoA OTU2 accounted for a high proportion of the comammox community. Based on this trend, the decrease in $\mathrm{NH}_{3}$ concentration caused by the $\mathrm{pH}$ change on day 374 would not be critical to the nitrification activity of soil Nitrospira amoA OTU2. Indeed, soil Nitrospira amoA OTU2 was unable to grow under $\mathrm{pH} 5.5$ like typical ammonia oxidizers (De Boer and Kowalchuk, 2001). Instead, soil Nitrospira amoA OTU1 became dominant under relatively acidic conditions at $\mathrm{pH}$ 5.5.

Moreover, unlike the dynamic change in the comammox amoA gene-based community structure, 16S rRNA gene-based analysis of the Nitrospira community showed no significant change before and after the $\mathrm{pH}$ decrease (Figure 3C). The gap between these two analyses was considered to be caused by the difference in the similarities of nucleotide sequences. Partial $16 \mathrm{~S}$ rRNA gene sequences of nitrifiers have high similarities, and thus, it is difficult to distinguish between closely related bacteria. In contrast, functional marker genes are considered to be suitable for analyzing the diversity in detail and characterizing the phenotypic features (Norton et al., 2002). Especially, the amo $A$ gene has been used as a suitable functional marker gene to analyze the phylogeny of comammox Nitrospira because it enables comammox and nitrite-oxidizing Nitrospira to be differentiated (Yu et al., 2018). In this study, analysis based on the amoA gene sequence detected several groups of comammox Nitrospira. Generally, genomes of comammox Nitrospira harbor one single copy of the amoA gene (Palomo et al., 2018). Therefore, several types of comammox were presumed to exist in the bioreactor. Even though amoA gene sequences detected in this experiment were classified into different OTUs, amplicon sequencing of the $16 \mathrm{~S}$ rRNA gene found only one OTU in the $\mathrm{NH}_{4} \mathrm{Cl}$-fed bioreactor. This could be because the phylogenetically distinct types of comammox at the amoA gene level shared a high proportion of $16 \mathrm{~S}$ rRNA gene sequences, and they were grouped into an identical OTU. This impossibility of linking each amoA OTU with one specific 16S rRNA gene sequence could be solved by using a single-cell isolation system combined with the de novo assembly of single-cell genomes (Chitsaz et al., 2011). 


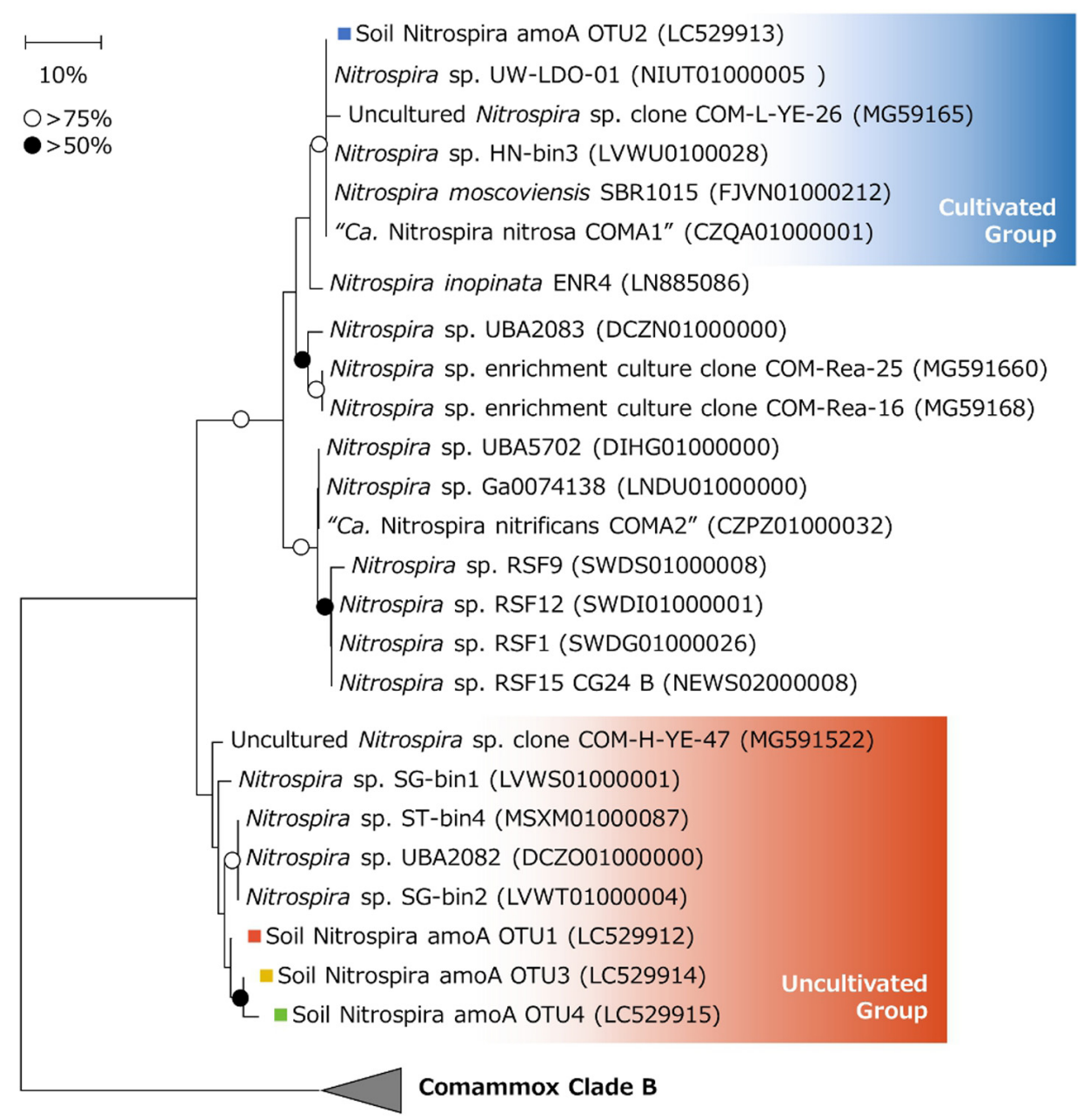

FIGURE 6 | Phylogenetic tree of comammox clade A based on AmoA amino acid sequences translated from nucleotide sequences. Phylogenetic analysis was performed using the neighbor-joining method with 1000 bootstraps. Bootstrap values of $>75 \%$ or $>50 \%$ are indicated as open or closed circles, respectively. Colored squares show the OTUs obtained in this study. Comammox clade B sequences (NEWT02000035, NEWR02000002, OEKW00000000, and OELE00000000) were used as outgroups.

\section{AmoA-Based Phylogenetic Analysis of Comammox Nitrospira}

The nucleotide sequences of comammox amoA OTUs were translated into amino acid sequences. A phylogenetic tree was constructed by referring to these translated sequences and those registered in the NCBI database. Comammox AmoA are known to be divided into two clades, and the primer pair used in this study was able to amplify the amoA genes of both clade A and B (Fowler et al., 2018). However, all of the detected OTUs were classified into clade A. Looking in further detail, soil Nitrospira amoA OTU2 and other OTUs were classified into different branches of clade A (Figure 6). The phylogenetical gap between these two groups corresponded to the difference in low $\mathrm{pH}$ adaptation; soil Nitrospira amoA OTU2 was deactivated at $\mathrm{pH}$ 5.5, while the other three OTUs survived (Table 1). Moreover, soil Nitrospira amoA OTU2 was grouped into the same branch with a cultivated comammox Nitrospira reported in previous studies (Daims et al., 2015; van Kessel et al., 2015; Camejo et al., 2017; Yu et al., 2018). On the other hand, soil Nitrospira amoA OTU1, OTU3, and OTU4 were classified into another branch with uncultured Nitrospira. Genes encoding AmoA of those Nitrospira were detected in drinking water (Wang et al., 2017), marine sediments (Parks et al., 2017) and river sediments (Yu et al., 2018). In the study cultivating sediments by $\mathrm{Yu}$ et al., uncultured Nitrospira sp. Clone COM-H-YE47 was found. Interestingly, however, no identical amo $A$ gene sequence was detected in a $\mathrm{NH}_{4} \mathrm{Cl}$-fed bioreactor incubating the sediment sample (Yu et al., 2018). From these results, it could be inferred that the continuous-feeding bioreactor in this study enabled the enrichment of a group of comammox Nitrospira that were fastidious and not cultivated in previous studies. Furthermore, these enriched comammox Nitrospira were not only physiologically novel, but also phylogenetically distinct from the strains cultivated in previous researches. 


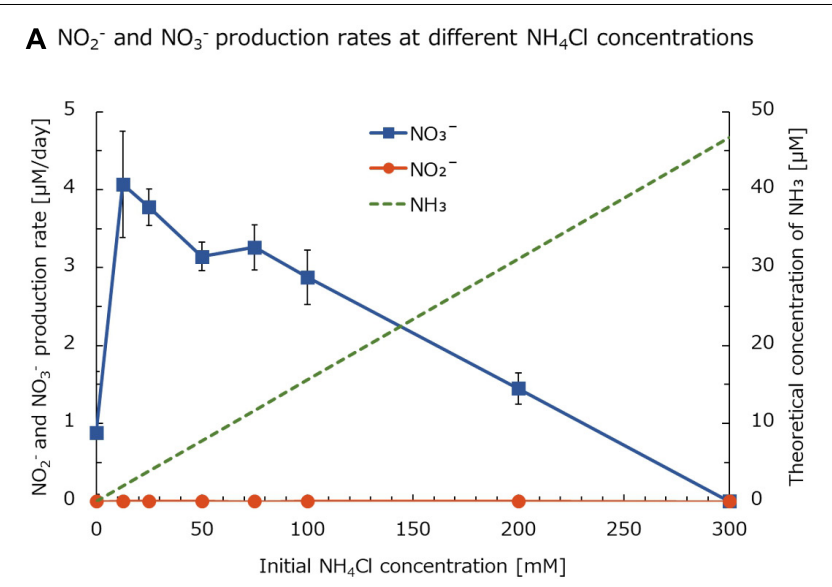

B $\quad \mathrm{NO}_{2}^{-}$and $\mathrm{NO}_{3}{ }^{-}$production rates at different $\mathrm{pH}$ values

FIGURE 7 | (A) $\mathrm{NO}_{2}{ }^{-}$and $\mathrm{NO}_{3}{ }^{-}$production rates of the enrichment sample from the $\mathrm{NH}_{4} \mathrm{Cl}$-fed bioreactor at different $\mathrm{NH}_{4} \mathrm{Cl}$ concentrations. The initial pH of media was adjusted to 5.5. (B) $\mathrm{NO}_{2}{ }^{-}$and $\mathrm{NO}_{3}-$ production rates of the enrichment sample from the $\mathrm{NH}_{4} \mathrm{Cl}$-fed bioreactor at different $\mathrm{pH}$ values. The medium was supplemented with $12.5 \mathrm{mM}$ of $\mathrm{NH}_{4} \mathrm{Cl}$. Data show means and error bars show standard deviation of biological triplicates. Green dashed lines show the theoretically calculated concentrations of $\mathrm{NH}_{3}$.

\section{Effect of the $\mathrm{NH}_{4} \mathrm{Cl}$ Concentration and $\mathrm{pH}$ on the Nitrification Activity of the Enrichment Sample}

To evaluate the nitrification activity of the enrichment sample in the $\mathrm{NH}_{4} \mathrm{Cl}$-fed bioreactor, the culture sample was collected on day 749 and the activity was tested under different $\mathrm{NH}_{4} \mathrm{Cl}$ concentrations. The initial $\mathrm{pH}$ of the medium was adjusted to $\mathrm{pH} 5.5$, the same value as the medium in the bioreactor. Furthermore, the $\mathrm{NH}_{4} \mathrm{Cl}$ concentration in the medium was adjusted at $0,12.5,25,50,75,100,200$, or $300 \mathrm{mM}$. After three days of incubation, $\mathrm{NO}_{3}{ }^{-}$was accumulated in the samples, but $\mathrm{NO}_{2}^{-}$was rarely detected (Figure $7 \mathbf{A}$ ). This result indicated that all the oxidized ammonia was converted to nitrate. Even in the sample not supplemented with $\mathrm{NH}_{4} \mathrm{Cl}, \mathrm{NO}_{3}{ }^{-}$was slightly generated. This is likely because a small amount of ammonium was produced by the degradation of organic nitrogen such as cyanate or urea. Considering the culture sample containing heterotrophic bacteria other than chemolithoautotrophic nitrifiers, the amount of organic nitrogen generated from the biomass could not be negligible.

The $\mathrm{NO}_{3}{ }^{-}$production rate was the highest at a $\mathrm{NH}_{4} \mathrm{Cl}$ concentration of $12.5 \mathrm{mM}$ (Figure 7A). The theoretical concentration of free ammonia $\left(\mathrm{NH}_{3}\right)$ under this condition was calculated to be $1.94 \mu \mathrm{M}$. This value is smaller than $33.3 \mu \mathrm{M}$, the half saturation constant $\left(K_{\mathrm{m}}\right)$ of $\mathrm{NH}_{3}$ reported for "Ca. Nitrosoglobus terrae", $\mathrm{AOB}$ isolated from acidic soil (Hayatsu et al., 2017). On the other hand, AOA isolated from acidic soil, "Ca. Nitrosotalea devanaterra", showed the highest growth rate with $0.18 \mathrm{nM} \mathrm{NH}_{3}$ (Lehtovirta-Morley et al., 2011). Therefore, the optimum $\mathrm{NH}_{3}$ concentration for comammox Nitrospira enriched in this study would be higher than that for AOA but lower than that for AOB cultured from acidic soils. Based on these results, the niche differentiation among terrestrial comammox

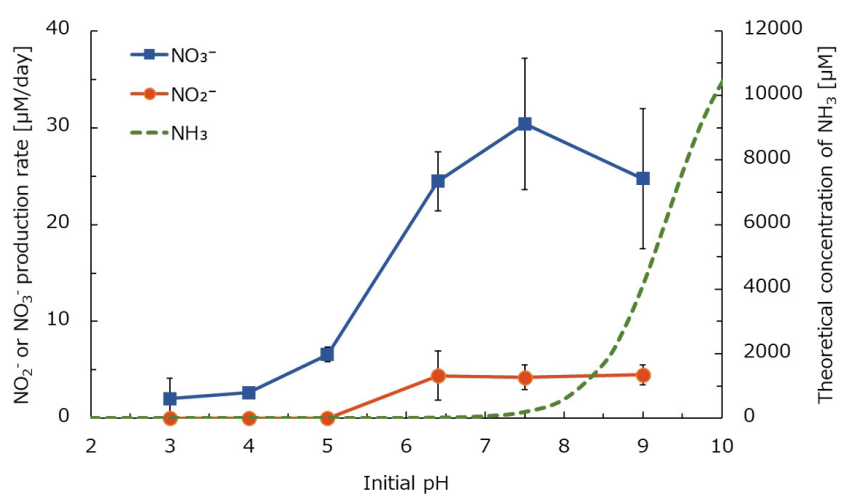

Nitrospira, AOB, and AOA is considered to partly depend on a favorable $\mathrm{NH}_{3}$ concentration. This trend corresponds to the difference in affinity for $\mathrm{NH}_{3}$, described in a previous study testing the physiological characteristics of Nitrospira inopinata (Dimitri et al., 2017). Moreover, the $K_{\mathrm{m}}$ value of $\mathrm{NH}_{3}$ reported for Nitrospira inopinata is around 49 to $83 \mathrm{nM}$ (Dimitri et al., 2017). Although this value is lower than $1.94 \mu \mathrm{M}$, the optimum concentration for the enrichment in this study, it is still larger than that of terrestrial AOA.

Furthermore, the nitrification rate of the enrichment from the $\mathrm{NH}_{4} \mathrm{Cl}$-fed bioreactor decreased as the initial $\mathrm{NH}_{4} \mathrm{Cl}$ concentration increased (Figure 7A). The enrichment was still active at $200 \mathrm{mM} \mathrm{NH} \mathrm{m}_{4} \mathrm{Cl}$. However, the nitrification activity was inhibited by $300 \mathrm{mM} \mathrm{NH} \mathrm{N}_{4} \mathrm{Cl}$, which was equivalent to $46.7 \mu \mathrm{M} \mathrm{NH}$ at $\mathrm{pH}$ 5.5. This value almost matches the $K_{\mathrm{m}}$ of $\mathrm{NH}_{3}$ for "Ca. Nitrosoglobus terrae", as described above (Hayatsu et al., 2017). Considering that an acid-adapted AOB could tolerate this amount of $\mathrm{NH}_{3}$, the nitrification at $\mathrm{pH} 5.5$ in this experiment was mainly performed by comammox rather than $\mathrm{AOB}$, although the inhibition of comammox Nitrospira caused by a high concentration of $\mathrm{NH}_{3}$ has not been reported by physiological experiments with pure cultures.

Moreover, to investigate the adaptation to $\mathrm{pH}$ change, the nitrification activity of the enrichment sample from the $\mathrm{NH}_{4} \mathrm{Cl}$-fed bioreactor was tested under a variety of $\mathrm{pH}$ values. After 2 days of incubation, the decrease in $\mathrm{pH}$ was kept within 1.0 in all samples (data not shown). The enrichment sample oxidized $\mathrm{NH}_{3}$ to $\mathrm{NO}_{3}{ }^{-}$at a wide range of $\mathrm{pH}$ values (3.0-9.0). Within $\mathrm{pH}$ 6.4-9.0, $\mathrm{NH}_{3}$ was not completely oxidized and $\mathrm{NO}_{2}{ }^{-}$was accumulated in the culture (Figure 7B). However, at this $\mathrm{pH}$ range, $\mathrm{NH}_{3}$ was oxidized relatively faster than at $\mathrm{pH}$ 3.0-5.0. Under higher $\mathrm{pH}$ conditions, the concentration of $\mathrm{NH}_{3}$ 
increases because of deprotonation. Especially at $\mathrm{pH} 7.5$ and 9.0, the theoretical concentration of $\mathrm{NH}_{3}$ was 192 and 4,122 $\mu \mathrm{M}$, respectively. These values are much higher than the $46.7 \mu \mathrm{M}$ inhibiting the nitrification activity of the enrichment at $\mathrm{pH} 5.5$ (Figure 7A). Based on these results, the nitrifier oxidizing $\mathrm{NH}_{3}$ to $\mathrm{NO}_{3}^{-}$at $\mathrm{pH}$ 7.59.0 would be different from the major nitrifier oxidizing $\mathrm{NH}_{3}$ at $\mathrm{pH} 5.5$.

According to the $16 \mathrm{~S}$ rRNA gene-based analysis, betaproteobacterial AOB enriched in the bioreactor was closely related to Nitrosospira lacus. Nitrosospira lacus was reported to oxidize $\mathrm{NH}_{3}$ under a broad range of $\mathrm{pH}$ values (5-9) (Urakawa et al., 2015). Furthermore, the $\mathrm{NH}_{3}$ oxidation activity of Nitrosospira lacus sharply decreased at $\mathrm{pH} 6$ (Ishii et al., 2017). This trend corresponds to the low $\mathrm{NO}_{3}{ }^{-}$ production rate at $\mathrm{pH} 3.0-5.0$ in this study (Figure $7 \mathbf{B}$ ). On the other hand, the slow $\mathrm{NO}_{3}{ }^{-}$production at $\mathrm{pH}$ 3.0-5.0 would be carried out by comammox Nitrospira. The $\mathrm{NO}_{3}{ }^{-}$ production rate in this $\mathrm{pH}$ range was almost equivalent to the rate at $\mathrm{pH} 5.5$ measured in the former experiment (Figure 7A). Therefore, $\mathrm{NH}_{3}$ might be mainly oxidized by comammox Nitrospira and Nitrosospira at $\mathrm{pH} 3.0-5.0$ and 6.4-9.0, respectively. Thus, the gap in the $\mathrm{NH}_{3}$ oxidation rate between these two $\mathrm{pH}$ ranges might be caused by the difference in the maximum oxidation rate between AOB and comammox Nitrospira. According to a previous report, the maximum rate of ammonia oxidation of Nitrosomonas europaea, a strain of betaproteobacterial AOB, was several times higher than that of Nitrospira inopinata (Dimitri et al., 2017). From these results, the rapid $\mathrm{NH}_{3}$ oxidation at $\mathrm{pH}$ 6.4-9.0 was considered to be caused by AOB, although the relative abundance of Nitrosospira was lower than that of Nitrospira in the enrichment sample. Within $\mathrm{pH}$ 6.4-9.0, $\mathrm{NO}_{2}{ }^{-}$was accumulated in the medium, which also supports two-step oxidation from $\mathrm{NH}_{3}$ to $\mathrm{NO}_{3}{ }^{-}$via $\mathrm{NO}_{2}{ }^{-}$by $\mathrm{AOB}$ and $\mathrm{NOB}$. Considering the accumulation of $\mathrm{NO}_{2}{ }^{-}$in this experiment, the NOB activity in the enrichment sample might not be sufficient to completely oxidize $\mathrm{NO}_{2}{ }^{-}$. This could be partly because the abundance of Nitrobacter was lower than that of Nitrosospira in the enrichment sample. On the other hand, $\mathrm{NO}_{2}{ }^{-}$was not detected at $\mathrm{pH} 3.0-5.0$. This result suggests that the complete nitrification occurred at $\mathrm{pH}$ 3.0-5.0. However, $\mathrm{NO}_{2}{ }^{-}$can be converted to $\mathrm{NO}_{3}{ }^{-}$abiotically at $\mathrm{pH}<3.3$ (Cai et al., 2001). Thus, the complete nitrification at $\mathrm{pH} 3.0$ is still debatable.

In this study, $\mathrm{NH}_{3}$ oxidation at $\mathrm{pH} 3.0-5.0$ seemed to be carried out by comammox Nitrospira. According to previous studies, ammonia oxidizers cultured from acidic soils, " $\mathrm{Ca}$. Nitrosoglobus terrae" and "Ca. Nitrosotalea devanaterra", were unable to oxidize $\mathrm{NH}_{3}$ at $\mathrm{pH}$ 3.5. From these results, comammox Nitrospira enriched in this study would have an equal or higher tolerance to low $\mathrm{pH}$ as acid-adapted $\mathrm{AOB}$ and AOA. Even though the contribution of each microbe to the nitrification in this experiment was still unclear, this study gives evidence of the ability of diverse nitrifying bacteria communities in soil to adapt to a wide range of $\mathrm{pH}$ values.

\section{CONCLUSION}

In this study, we selectively enriched both comammox and nitrite-oxidizing Nitrospira from acidic soils of a tea field fertilized with a large amount of nitrogen. A phylogenetical analysis based on the 16S rRNA gene revealed the niche differentiation between nitrite-oxidizing Nitrospira lineage I and lineage II depending on the $\mathrm{HNO}_{2}$ concentration rather than $\mathrm{pH}$. Moreover, the amplicon sequencing targeting the amoA gene detected OTUs related to uncultivated comammox Nitrospira. The enrichment sample of comammox Nitrospira showed nitrification activity even at $\mathrm{pH}$ 3-4. Therefore, the enriched comammox Nitrospira was not only phylogenetically novel, but also might possess the potential to adapt to low $\mathrm{pH}$.

Although Nitrospira, as either comammox or NOB, is still difficult to cultivate, physiological studies are necessary to understand their characteristics. This study provides a scientific basis to establish a cultivation method for Nitrospira from acidic soils. Furthermore, the enrichment sample enables us to examine the metabolism of Nitrospira by comprehensive analyses based on metatranscriptomics or metaproteomics. These approaches could reveal the still unknown acid-adaptation mechanisms of Nitrospira and the regulation factors that affect these systems.

\section{DATA AVAILABILITY STATEMENT}

The amplicon sequence data have been deposited in DDBJ Sequence Read Archive with BioProject number PRJDB9437. The OTUs acquired from amplicon sequencing based on the 16S rRNA gene and amoA gene were registered at DDBJ/ENA/GenBank with accession numbers LC529906, LC529907, LC529908, LC529909, LC529910, LC529911, LC529912, LC529913, LC529914, and LC529915.

\section{AUTHOR CONTRIBUTIONS}

YT, HF, and ST contributed to the conception and design of the study, analyzed the phylogenetical and physiological data, and wrote the manuscript with help from all coauthors. YT cultivated the soil sample and performed all of the experiments using enrichment cultures. $\mathrm{YH}$ collected the soil sample. KT, YW, and $\mathrm{MH}$ analyzed and identified the chemical characteristics of the soil sample. All authors contributed to the article and approved the submitted version.

\section{FUNDING}

This study was supported by the Japan Society for the Promotion of Science with KAKENHI (19K16218) (to $\mathrm{HF}$ ), the Mayekawa Houonkai Foundation with Scientific Research Grant (A16034) (to HF), and the Waseda 
University Grant for Special Research Projects (2019C-275) for supporting this research.

\section{ACKNOWLEDGMENTS}

We thank Dr. Toshi Urakawa (Florida Gulf Coast University, Fort Myers, FL, United States) for offering advice to this research. We also would like to thank Kento Ishii for scientific discussion.

\section{REFERENCES}

Amann, R. I., Krumholz, L., and Stahl, D. A. (1990). Fluorescent-oligonucleotide probing of whole cells for determinative, phylogenetic, and environmental studies in microbiology. J. Bacteriol. 172, 762-770. doi: 10.1128/jb.172.2.762770.1990

Aminot, A., Kirkwood, D. S., and Kérouel, R. (1997). Determination of ammonia in seawater by the indolphenol-blue method: evaluation of the ICES NUTS I/C 5 questionnaire. Mar. Chem. 56, 59-75. doi: 10.1016/s0304-4203(96)00080-1

Anthonisen, A. C., Loehr, R. C., Prakasam, T. B. S., and Srinath, E. G. (1976). Inhibition of nitrification by ammonia and nitrous acid. J. Water Pollut. Control Federation 48, 835-852.

Bakken, L. R., Bergaust, L., Liu, B., and Frostegård, ^̊ (2012). Regulation of denitrification at the cellular level: a clue to the understanding of $\mathrm{N} 2 \mathrm{O}$ emissions from soils. Philos. Trans. R. Soc. BBiol. Sci. 367, 1226-1234. doi: 10.1098/rstb. 2011.0321

Bartelme, R. P., McLellan, S. L., and Newton, R. J. (2017). Freshwater recirculating aquaculture system operations drive biofilter bacterial community shifts around a stable nitrifying consortium of ammonia-oxidizing archaea and comammox Nitrospira. Front. Microbiol. 8:101. doi: 10.3389/fmicb.2017.00101

Blackburne, R., Vadivelu, V. M., Yuan, Z., and Keller, J. (2007). Kinetic characterisation of an enriched Nitrospira culture with comparison to Nitrobacter. Water Res. 41, 3033-3042. doi: 10.1016/j.watres.2007.01.043

Bock, E., and Heinrich, G. (1969). Morphological and physiological studies of Nitrobacter winogradskyi Buch. Archiv. Mikrobiolo. 69, 19-59.

Booth, M. S., Stark, J. M., and Rastetter, E. (2005). Controls on nitrogen cycling in terrestrial ecosystems: a synthetic analysis of literature data. Ecol. Monogr. 75, 139-157. doi: 10.1890/04-0988

Cai, Q., Zhang, W., and Yang, Z. (2001). Stability of nitrite in wastewater and its determination by ion chromatography. Anal. Sci. 17, 917-920.

Camejo, P. Y., Domingo, J. S., McMahon, K. D., and Noguera, D. R. (2017). Genome-enabled insights into the ecophysiology of the comammox bacterium "candidatus Nitrospira nitrosa". mSystems 2:e00059-17. doi: 10.1128/mSystems. 00059-17

Caporaso, J. G., Kuczynski, J., Stombaugh, J., Bittinger, K., Bushman, F. D., Costello, E. K., et al. (2010). QIIME allows analysis of high-throughput community sequencing data. Nat. Methods 7, 335-336.

Chitsaz, H., Yee-Greenbaum, J. L., Tesler, G., Lombardo, M. J., Dupont, C. L., Badger, J. H., et al. (2011). Efficient de novo assembly of single-cell bacterial genomes from short-read data sets. Nat. Biotechnol. 29, 915-921 . doi: 10.1038/ nbt.1966

Cytryn, E., Levkovitch, I., Negreanu, Y., Dowd, S., Frenk, S., and Silber, A. (2012). Impact of short-term acidification on nitrification and nitrifying bacterial community dynamics in soilless cultivation media. Appl. Environ. Microbiol. 78, 6576-6582. doi: 10.1128/aem.01545-12

Dai, G. Z., Shang, J. L., and Qiu, B. S. (2012). Ammonia may play an important role in the succession of cyanobacterial blooms and the distribution of common algal species in shallow freshwater lakes. Global Change Biol. 18, 1571-1581. doi: 10.1111/j.1365-2486.2012.02638.x

Daims, H., Lebedeva, E. V., Pjevac, P., Han, P., Herbold, C., Albertsen, M., et al. (2015). Complete nitrification by Nitrospira bacteria. Nature 528, 504-509. doi: 10.1038/nature16461

De Boer, W., Gunnewiek, P. J. A. K., Veenhuis, M., Bock, E., and Laanbroek, H. J. (1991). Nitrification at low $\mathrm{pH}$ by aggregated chemolithotrophic bacteria. Appl. Environ. Microbiol. 57, 3600-3604. doi: 10.1128/aem.57.12.3600-3604.1991
Furthermore, we would like to thank Editage (www.editage.com) for English language editing.

\section{SUPPLEMENTARY MATERIAL}

The Supplementary Material for this article can be found online at: https://www.frontiersin.org/articles/10.3389/fmicb. 2020.01737/full\#supplementary-material

De Boer, W., and Kowalchuk, G. A. (2001). Nitrification in acid soils: microorganisms and mechanisms. Soil Biol. Biochem. 33, 853-866. doi: 10.1016/ s0038-0717(00)00247-9

Dimitri, K., Sedlacek, C. J., Lebedeva, E. V., Han, P., Bulaev, A., Pjevac, P., et al. (2017). Kinetic analysis of a complete nitrifier reveals an oligotrophic lifestyle. Nature 549, 269-272. doi: 10.1038/nature23679

Edgar, R. C. (2010). Search and clustering orders of magnitude faster than BLAST. Bioinformatics 26, 2460-2461. doi: 10.1093/bioinformatics/btq461

Felsenstein, J. (1985). Confidence limits on phylogenies: an approach using the bootstrap. Evolution 39, 783-791. doi: 10.1111/j.1558-5646.1985.tb00420.x

Fowler, S. J., Palomo, A., Dechesne, A., Mines, P. D., and Smets, B. F. (2018). Comammox Nitrospira are abundant ammonia oxidizers in diverse groundwater-fed rapid sand filter communities. Environ. Microbiol. 20, 10021015. doi: 10.1111/1462-2920.14033

Fujitani, H., Aoi, Y., and Tsuneda, S. (2013). Selective enrichment of two different types of Nitrospira-like nitrite-oxidizing bacteria from a wastewater treatment plant. Microb. Environ. 28, 236-243. doi: 10.1264/jsme2.me12209

Galloway, J. N., Townsend, A. R., Erisman, J. W., Bekunda, M., Cai, Z., Freney, J. R., et al. (2008). Transformation of the nitrogen cycle: recent trends, questions, and potential solutions. Science 320, 889-892. doi: 10.1126/science.1136674

Garcia, J. C., Urakawa, H., Le, V. Q., Stein, L. Y., Klotz, M. G., and Nielsen, J. L. (2013). Draft genome sequence of Nitrosospira sp. strain APG3, a psychrotolerant ammonia-oxidizing bacterium isolated from sandy lake sediment. Genome Announc. 1:e00930-13. doi: 10.1128/genomeA.00930-13

Griess-Romijn, E. (1966). Physiological and Chemical Tests for Drinking Water, in NEN 1056, IV-2. Rijswijk: Nederlands Normalisatie Instituut.

Gruber-Dorninger, C., Pester, M., Kitzinger, K., Savio, D. F., Loy, A., Rattei, T., et al. (2015). Functionally relevant diversity of closely related Nitrospira in activated sludge. ISME J. 9, 643-655. doi: 10.1038/ismej.2014.156

Hankinson, T. R., and Schmidt, E. L. (1988). An acidophilic and a neutrophilic Nitrobacter strain isolated from the numerically predominant nitrite-oxidizing population of an acid forest soil. Appl. Environ. Microbiol. 54, 1536-1540. doi: 10.1128/aem.54.6.1536-1540.1988

Hayatsu, M., Tago, K., Uchiyama, I., Toyoda, A., Wang, Y., Shimomura, Y., et al. (2017). An acid-tolerant ammonia-oxidizing $\gamma$-proteobacterium from soil. ISME J. 11, 1130-1141. doi: 10.1038/ismej.2016.191

Herbold, C. W., Lehtovirta-Morley, L. E., Jung, M. Y., Jehmlich, N., Hausmann, B., Han, P., et al. (2017). Ammonia-oxidising archaea living at low pH: insights from comparative genomics. Environ. Microbiol. 19, 4939-4952. doi: 10.1111/ 1462-2920.13971

Hirono, Y., Watanabe, I., and Nonaka, K. (2009). Trends in water quality around an intensive tea-growing area in Shizuoka. Jpn. Soil Sci. Plant Nutr. 55, 783-792. doi: 10.1111/j.1747-0765.2009.00413.x

Hu, H. W., and He, J. Z. (2017). Comammox-a newly discovered nitrification process in the terrestrial nitrogen cycle. J. Soils and Sediments 17, 2709-2717. doi: 10.1007/s11368-017-1851-9

Ishii, K., Fujitani, H., Soh, K., Nakagawa, T., Takahashi, R., and Tsuneda, S. (2017). Enrichment and physiological characterization of a coldadapted nitriteoxidizing Nitrotoga sp. from an eelgrass sediment. Appl. Environ. Microbiol. 83:AEM.00549-17. doi: 10.1128/AEM.00549-17

Kandeler, E., and Gerber, H. (1988). Short-term assay of soil urease activity using colorimetric determination of ammonium. Biol. Fertil. Soils 6, 68-72.

Kérouel, R., and Aminot, A. (1997). Fluorometric determination of ammonia in sea and estuarine waters by direct segmented flow analysis. Mar. Chem. 57, 265-275. doi: 10.1016/s0304-4203(97)00040-6 
Koch, H., van Kessel, M., and Lücker, S. (2019). Complete nitrification: insights into the ecophysiology of comammox Nitrospira. Appl. Microbiol. Biotechnol. 103, 177-189. doi: 10.1007/s00253-018-9486-3

Koper, T. E., El-Sheikh, A. F., Norton, J. M., and Klotz, M. G. (2004). Ureaseencoding genes in ammonia-oxidizing bacteria. Appl. Environ. Microbiol. 70, 2342-2348. doi: 10.1128/aem.70.4.2342-2348.2004

Kostka, J., Green, S., Rishishwar, L., Om Prakash Katz, L., Mariño-Ramírez, L., Jordan, K., et al. (2012). Genome sequences for six rhodanobacter strains, isolated from soils and the terrestrial subsurface, with variable denitrification capabilities. J. Bacteriol. 194, 4461-4462. doi: 10.1128/jb.00871-12

Kowalchuk, G. A., and Stephen, J. R. (2001). Ammonia-oxidizing bacteria: a model for molecular microbial ecology. Annu. Rev. Microbiol. 55, 485-529. doi: 10. 1146/annurev.micro.55.1.485

Laanbroek, H. J., and Schotman, J. M. T. (1991). Effect of nitrite concentration and $\mathrm{pH}$ on most probable number enumerations of non-growing Nitrobacter spp. FEMS Microbiol. Ecol. 8, 269-277. doi: 10.1111/j.1574-6941.1991.tb0 1772.x

Lehtovirta-Morley, L. E., Ge, C., Ross, J., Yao, H., Nicol, G. W., and Prosser, J. I. (2014). Characterisation of terrestrial acidophilic archaeal ammonia oxidisers and their inhibition and stimulation by organic compounds. FEMS Microbiol. Ecol. 89, 542-552. doi: 10.1111/1574-6941.12353

Lehtovirta-Morley, L. E., Sayavedra-Soto, L. A., Gallois, N., Schouten, S., Stein, L. Y., Prosser, J. I., et al. (2016). Identifying potential mechanisms enabling acidophily in the ammonia-oxidizing archaeon "Candidatus Nitrosotalea devanaterra”. Appl. Environ. Microbiol. 82, 2608-2619. doi: 10.1128/aem. 04031- 15

Lehtovirta-Morley, L. E., Stoecker, K., Vilcinskas, A., Prosser, J. I., and Nicol, G. W. (2011). Cultivation of an obligate acidophilic ammonia oxidizer from a nitrifying acid soil. Proc. Natl. Acad. Sci. U.S.A. 108, 15892-15897. doi: 10.1073/pnas.1107196108

Leininger, S., Urich, T., Schloter, M., Schwark, L., Qi, J., Nicol, G. W., et al. (2006). Archaea predominate among ammonia-oxidizing prokaryotes in soils. Nature 442, 806-809. doi: 10.1038/nature04983

Liu, B., Mørkved, P. T., Frostegård, A., and Bakken, L. R. (2010). Denitrification gene pools, transcription and kinetics of $\mathrm{NO}, \mathrm{N} 2 \mathrm{O}$ and $\mathrm{N} 2$ production as affected by soil pH. FEMS Microbiol. Ecol. 72, 407-417. doi: 10.1111/j.15746941.2010.00856.x

Long, X., Chen, C., Xu, Z., Oren, R., and He, J. Z. (2012). Abundance and community structure of ammonia-oxidizing bacteria and archaea in a temperate forest ecosystem under ten-years elevated CO2. Soil Biol. Biochem. 46, 163-171. doi: 10.1016/j.soilbio.2011.12.013

Maixner, F., Noguera, D. R., Anneser, B., Stoecker, K., Wegl, G., Wagner, M., et al. (2006). Nitrite concentration influences the population structure of Nitrospiralike bacteria. Environ. Microbiol. 8, 1487-1495. doi: 10.1111/j.1462-2920.2006. 01033.x

McDonald, D., Price, M. N., Goodrich, J., Nawrocki, E. P., DeSantis, T. Z., Probst, A., et al. (2012). An improved Greengenes taxonomy with explicit ranks for ecological and evolutionary analyses of bacteria and archaea. ISME J. 6, 610-618. doi: 10.1038/ismej.2011.139

Miranda, K. M., Espey, M. G., and Wink, D. A. (2001). A rapid, simple spectrophotometric method for simultaneous detection of nitrate and nitrite. Nitric Oxide Biol. Chem. 5, 62-71. doi: 10.1006/niox.2000.0319

Mørkved, P. T., Dörsch, P., and Bakken, L. R. (2007). The N2O product ratio of nitrification and its dependence on long-term changes in soil pH. Soil Biol. Biochem. 39, 2048-2057. doi: 10.1016/j.soilbio.2007.03.006

Nicol, G. W., Leininger, S., Schleper, C., and Prosser, J. I. (2008). The influence of soil $\mathrm{pH}$ on the diversity, abundance and transcriptional activity of ammonia oxidizing archaea and bacteria. Environ. Microbiol. 10, 2966-2978. doi: 10. 1111/j.1462-2920.2008.01701.x

Norton, J. M., Alzerreca, J. J., Suwa, Y., and Klotz, M. G. (2002). Diversity of ammonia monooxygenase operon in autotrophic ammonia-oxidizing bacteria. Arch. Microbiol. 177, 139-149. doi: 10.1007/s00203-001-0369-z

Norton, J. M., Klotz, M. G., Stein, L. Y., Arp, D. J., Bottomley, P. J., Chain, P. S. G., et al. (2008). Complete genome sequence of Nitrosospira multiformis, an ammonia-oxidizing bacterium from the soil environment. Appl. Environ. Microbiol. 74, 3559-3572. doi: 10.1128/aem.02722-07
Nowka, B., Off, S., Daims, H., and Spieck, E. (2015). Improved isolation strategies allowed the phenotypic differentiation of two Nitrospira strains from widespread phylogenetic lineages. FEMS Microbiol. Ecol. 91:fiu031.

Orellana, L. H., Chee-Sanford, J. C., Sanford, R. A., Löffler, F. E., and Konstantinidis, K. T. (2018). Year-round shotgun metagenomes reveal stable microbial communities in agricultural soils and novel ammonia oxidizers responding to fertilization. Appl. Environ. Microbiol. 84:e01646-17. doi: 10. 1128/AEM.01646-17

Palomo, A., Jane Fowler, S., Gülay, A., Rasmussen, S., Sicheritz-Ponten, T., and Smets, B. F. (2016). Metagenomic analysis of rapid gravity sand filter microbial communities suggests novel physiology of Nitrospira spp. ISME J. 10, 25692581. doi: $10.1038 /$ ismej.2016.63

Palomo, A., Pedersen, A. G., Fowler, S. J., Dechesne, A., Sicheritz-Pontén, T., and Smets, B. F. (2018). Comparative genomics sheds light on niche differentiation and the evolutionary history of comammox Nitrospira. ISME J. 12, 1779-1793. doi: 10.1038/s41396-018-0083-3

Park, H. D., and Noguera, D. R. (2008). Nitrospira community composition in nitrifying reactors operated with two different dissolved oxygen levels. J. Microbiol. Biotechnol. 18, 1470-1474.

Parks, D. H., Rinke, C., Chuvochina, M., Chaumeil, P. A., Woodcroft, B. J., Evans, P. N., et al. (2017). Recovery of nearly 8,000 metagenome-assembled genomes substantially expands the tree of life. Nat. Microbiol. 2, 1533-1542. doi: 10.1038/s41564-017-0012-7

Petersen, D. G., Blazewicz, S. J., Firestone, M., Herman, D. J., Turetsky, M., and Waldrop, M. (2012). Abundance of microbial genes associated with nitrogen cycling as indices of biogeochemical process rates across a vegetation gradient in Alaska. Environ. Microbiol. 14, 993-1008. doi: 10.1111/j.1462-2920.2011. 02679.x

Pjevac, P., Schauberger, C., Poghosyan, L., Herbold, C. W., van Kessel, M. A. H. J., Daebeler, A., et al. (2017). AmoA-targeted polymerase chain reaction primers for the specific detection and quantification of comammox Nitrospira in the environment. Front. Microbiol. 8:1508. doi: 10.3389/fmicb.2017.01508

Pommerening-Roser, A., and Koops, H. P. (2005). Environmental pH as an important factor for the distribution of urease positive ammonia-oxidizing bacteria. Microbiol. Res. 160, 27-35. doi: 10.1016/j.micres.2004.09.006

Quast, C., Pruesse, E., Yilmaz, P., Gerken, J., Schweer, T., Yarza, P., et al. (2013). The SILVA ribosomal RNA gene database project: improved data processing and web-based tools. Nucleic Acids Res. 41, D590-D596.

Reshetilov, A. N., Iliasov, P. V., Knackmuss, H. J., and Boronin, A. M. (2011). The nitrite oxidizing activity of nitrobacter strains as a base of microbial biosensor for nitrite detection. Anal. Lett. 33, 29-41. doi: 10.1080/00032710008543034

Roots, P., Wang, Y., Rosenthal, A. F., Griffin, J. S., Sabba, F., Petrovich, M., et al. (2019). Comammox Nitrospira are the dominant ammonia oxidizers in a mainstream low dissolved oxygen nitrification reactor. Water Res. 157, 396-405. doi: 10.1016/j.watres.2019.03.060

Saitou, N., and Nei, M. (1987). The neighbor-joining method: a new method for reconstructing phylogenetic trees. Mol. Biol. Evol. 4, 406-425.

Schlesinger, W. H. (2009). On the fate of anthropogenic nitrogen. Proc. Natl. Acad. Sci. U.S.A. 106, 203-208. doi: 10.1073/pnas.0810193105

Seemann, T. (2014). Prokka: rapid prokaryotic genome annotation. Bioinformatics 30, 2068-2069. doi: 10.1093/bioinformatics/btu153

Shindo, J., Okamoto, K., and Kawashima, H. (2006). Prediction of the environmental effects of excess nitrogen caused by increasing food demand with rapid economic growth in eastern Asian countries, 1961-2020. Ecol. Modelling 193, 703-720. doi: 10.1016/j.ecolmodel.2005.09.010

Spieck, E., Hartwig, C., McCormack, I., Maixner, F., Wagner, M., Lipski, A., et al. (2006). Selective enrichment and molecular characterization of a previously uncultured Nitrospira-like bacterium from activated sludge. Environ. Microbiol. 8, 405-415. doi: 10.1111/j.1462-2920.2005.00905.x

Stempfhuber, B., Richter-Heitmann, T., Bienek, L., Schöning, I., Schrumpf, M., Friedrich, M., et al. (2017). Soil pH and plant diversity drive co-occurrence patterns of ammonia and nitrite oxidizer in soils from forest ecosystems. Biol. Fertil. Soils 53, 691-700. doi: 10.1007/s00374-017-1215-z

Suzuki, I., Dular, U., and Kwok, S. C. (1974). Ammonia or ammonium ion as substrate for oxidation by Nitrosomonas europaea cells and extracts. J. Bacteriol. 120, 556-558. doi: 10.1128/jb.120.1.556-558.1974 
Tarre, S., and Green, M. (2004). High-rate nitrification at low $\mathrm{pH}$ in suspendedand attached-biomass reactors. Appl. Environ. Microbiol. 70, 6481-6487. doi: 10.1128/aem.70.11.6481-6487.2004

Tokuda, S., and Hayatsu, M. (2004). Nitrous oxide flux from a tea field amended with a large amount of nitrogen fertilizer and soil environmental factors controlling the flux. Soil Sci. Plant Nutr. 50, 365-374. doi: 10.1080/00380768. 2004.10408490

Tourna, M., Freitag, T. E., and Prosser, J. I. (2010). Stable isotope probing analysis of interactions between ammonia oxidizers. Appl. Environ. Microbiol. 76, 24682477. doi: 10.1128/aem.01964-09

Urakawa, H., Garcia, J. C., Nielsen, J. L., Le, V. Q., Kozlowski, J. A., Stein, L. Y., et al. (2015). Nitrosospira lacus sp. nov., a psychrotolerant, ammonia-oxidizing bacterium from sandy lake sediment. Int. J. Syst. Evol. Microbiol. 65, 242-250. doi: 10.1099/ijs.0.070789-0

Ushiki, N., Fujitani, H., Aoi, Y., and Tsuneda, S. (2013). Isolation of Nitrospira belonging to sublineage II from a wastewater treatment plant. Microbes Environ. 28, 346-353. doi: 10.1264/jsme2.me13042

van Kessel, M. A. H. J., Speth, D. R., Albertsen, M., Nielsen, P. H., Op Den Camp, H. J. M., Kartal, B., et al. (2015). Complete nitrification by a single microorganism. Nature 528, 555-559. doi: 10.1038/nature 16459

Vitousek, P. M., Aber, J. D., Howarth, R. W., Likens, G. E., Matson, P. A., Schindler, D. W., et al. (1997). Human alteration of the global nitrogen cycle: Sources and consequences. Ecol. Appl. 7, 737-750. doi: 10.1890/1051-0761(1997)007[0737: haotgn]2.0.co;2

Wang, Y., Ma, L., Mao, Y., Jiang, X., Xia, Y., Yu, K., et al. (2017). Comammox in drinking water systems. Water Res. 116, 332-341. doi: 10.1016/j.watres.2017. 03.042

Wang, Q., Quensen, J. F. III., Fish, J. A., Lee, T. K., Sun, Y., Tiedje, J. M., et al. (2013). Ecological patterns of nifH genes in four terrestrial climatic zones explored with targeted metagenomics using framebot, a new informatics tool. mBio 4:e00592-13. doi: 10.1128/mBio.00592-13

Wertz, S., Leigh, A. K., and Grayston, S. J. (2012). Effects of longterm fertilization of forest soils on potential nitrification and on the abundance and community structure of ammonia oxidizers and nitrite oxidizers. FEMS Microbiol. Ecol. 79, 142-154. doi: 10.1111/j.1574-6941.2011. 01204.x

Willason, S. W., and Johnson, K. S. (1986). A rapid, highly sensitive technique for the determination of ammonia in seawater. Mar. Biol. 91, 285-290. doi: 10.1007/bf00569445

Wrage, N., Velthof, G. L., Van Beusichem, M. L., and Oenema, O. (2001). Role of nitrifier denitrification in the production of nitrous oxide. Soil Biol. Biochem. 33, 1723-1732. doi: 10.1016/s0038-0717(01)00096-7

Yu, C., Hou, L., Zheng, Y., Liu, M., Yin, G., Gao, J., et al. (2018). Evidence for complete nitrification in enrichment culture of tidal sediments and diversity analysis of clade a comammox Nitrospira in natural environments. Appl. Microbiol. Biotechnol. 102, 9363-9377. doi: 10.1007/s00253-0189274-0

Zhang, J., Dai, Y., Wang, Y., Wu, Z., Xie, S., and Liu, Y. (2015). Distribution of ammonia-oxidizing archaea and bacteria in plateau soils across different land use types. Appl. Microbiol. Biotechnol. 99, 6899-6909. doi: 10.1007/s00253-0156625-y

Zhang, L. M., Hu, H. W., Shen, J. P., and He, J. Z. (2012). Ammoniaoxidizing archaea have more important role than ammonia-oxidizing bacteria in ammonia oxidation of strongly acidic soils. ISME J. 6, 1032-1045. doi: 10.1038/ismej.2011.168

Conflict of Interest: The authors declare that the research was conducted in the absence of any commercial or financial relationships that could be construed as a potential conflict of interest.

Copyright (c) 2020 Takahashi, Fujitani, Hirono, Tago, Wang, Hayatsu and Tsuneda. This is an open-access article distributed under the terms of the Creative Commons Attribution License (CC BY). The use, distribution or reproduction in other forums is permitted, provided the original author(s) and the copyright owner(s) are credited and that the original publication in this journal is cited, in accordance with accepted academic practice. No use, distribution or reproduction is permitted which does not comply with these terms. 